Louisiana State University

LSU Digital Commons

\title{
GRB 061121: Broadband spectral evolution through the prompt and afterglow phases of a bright burst
}

\author{
K. L. Page \\ University of Leicester \\ R. Willingale \\ University of Leicester \\ J. P. Osborne \\ University of Leicester \\ B. Zhang \\ University of Nevada, Las Vegas \\ O. Godet \\ University of Leicester
}

See next page for additional authors

Follow this and additional works at: https://digitalcommons.Isu.edu/physics_astronomy_pubs

\section{Recommended Citation}

Page, K., Willingale, R., Osborne, J., Zhang, B., Godet, O., Marshall, F., Melandri, A., Norris, J., O'Brien, P., Pal'shin, V., Rol, E., Romano, P., Starling, R., Schady, P., Yost, S., Barthelmy, S., Beardmore, A., Cusumano, G., Burrows, D., De Pasquale, M., Ehle, M., Evans, P., Gehrels, N., Goad, M., Golenetskii, S., Guidorzi, C., Mundell, C., Page, M., Ricker, G., Sakamoto, T., Schaefer, B., Stamatikos, M., \& Troja, E. (2007). GRB 061121: Broadband spectral evolution through the prompt and afterglow phases of a bright burst. Astrophysical Journal, 663 (2 I), 1125-1138. https://doi.org/10.1086/518821

This Article is brought to you for free and open access by the Department of Physics \& Astronomy at LSU Digital Commons. It has been accepted for inclusion in Faculty Publications by an authorized administrator of LSU Digital Commons. For more information, please contact ir@lsu.edu. 


\section{Authors}

K. L. Page, R. Willingale, J. P. Osborne, B. Zhang, O. Godet, F. E. Marshall, A. Melandri, J. P. Norris, P. T. O'Brien, V. Pal'shin, E. Rol, P. Romano, R. L.C. Starling, P. Schady, S. A. Yost, S. D. Barthelmy, A. P. Beardmore, G. Cusumano, D. N. Burrows, M. De Pasquale, M. Ehle, P. A. Evans, N. Gehrels, M. R. Goad, S. Golenetskii, C. Guidorzi, C. Mundell, M. J. Page, G. Ricker, T. Sakamoto, B. E. Schaefer, M. Stamatikos, and E. Troja 


\title{
GRB 061121: BROADBAND SPECTRAL EVOLUTION THROUGH THE PROMPT AND AFTERGLOW PHASES OF A BRIGHT BURST
}

\author{
K. L. Page, ${ }^{1}$ R. Willingale, ${ }^{1}$ J. P. Osborne, ${ }^{1}$ B. Zhang, ${ }^{2}$ O. Godet, ${ }^{1}$ F. E. Marshall,${ }^{3}$ A. Melandri, ${ }^{4}$ \\ J. P. Norris, ${ }^{5,6}$ P. T. O’Brien, ${ }^{1}$ V. Pal'shin, ${ }^{7}$ E. Rol, ${ }^{1}$ P. Romano, ${ }^{8,9}$ R. L. C. Starling, ${ }^{1}$ P. Schady, ${ }^{10}$ \\ S. A. Yost, ${ }^{11}$ S. D. Barthelmy, ${ }^{3}$ A. P. Beardmore, ${ }^{1}$ G. Cusumano, ${ }^{12}$ D. N. Burrows, ${ }^{13}$ \\ M. De Pasquale, ${ }^{10}$ M. Ehle, ${ }^{14}$ P. A. Evans, ${ }^{1}$ N. Gehrels, ${ }^{3}$ M. R. Goad,${ }^{1}$ \\ S. Golenetskit, ${ }^{7}$ C. Guidorzi, ${ }^{8,9}$ C. Mundell,${ }^{4}$ M. J. Page, ${ }^{10}$ G. Ricker, ${ }^{15}$ \\ T. Sakamoto, ${ }^{3}$ B. E. Schaefer, ${ }^{16}$ M. Stamatikos, ${ }^{3}$ E. Troja, ${ }^{1,12}$ \\ M. Ulanov, ${ }^{7}$ F. Yuan, ${ }^{11}$ and H. Ziaeepour ${ }^{9}$ \\ Received 2006 December 22; accepted 2007 April 12
}

\begin{abstract}
Swift triggered on a precursor to the main burst of GRB $061121(z=1.314)$, allowing observations to be made from the optical to gamma-ray bands. Many other telescopes, including Konus-Wind, XMM-Newton, ROTSE, and the Faulkes Telescope North, also observed the burst. The gamma-ray, X-ray, and UV/optical emission all showed a peak $\sim 75 \mathrm{~s}$ after the trigger, although the optical and X-ray afterglow components also appear early on, before or during the main peak. Spectral evolution was seen throughout the burst, with the prompt emission showing a clear positive correlation between brightness and hardness. The SED of the prompt emission, stretching from $1 \mathrm{eV}$ up to $1 \mathrm{MeV}$, is very flat, with a peak in the flux density at $\sim 1 \mathrm{keV}$. The optical to X-ray spectra at this time are better fitted by a broken, rather than single, power law, similar to previous results for X-ray flares. The SED shows spectral hardening as the afterglow evolves with time. This behavior might be a symptom of self-Comptonization, although circumstellar densities similar to those found in the cores of molecular clouds would be required. The afterglow also decays too slowly to be accounted for by the standard models. Although the precursor and main emission show different spectral lags, both are consistent with the lag-luminosity correlation for long bursts. GRB 061121 is the instantaneously brightest long burst yet detected by Swift. Using a combination of Swift and Konus-Wind data, we estimate an isotropic energy of $2.8 \times 10^{53}$ ergs over $1 \mathrm{keV}-10 \mathrm{MeV}$ in the GRB rest frame. A probable jet break is detected at $\sim 2 \times 10^{5} \mathrm{~s}$, leading to an estimate of $\sim 10^{51}$ ergs for the beaming-corrected gamma-ray energy.
\end{abstract}

Subject headings: gamma rays: bursts — X-rays: individual (GRB 061121)

\section{INTRODUCTION}

Gamma-ray bursts (GRBs) are intrinsically extremely luminous objects, approaching values of $10^{54} \mathrm{ergs} \mathrm{s}^{-1}$ if the radiation is isotropic (e.g., Frail et al. 2001; Bloom et al. 2003). This energy is emitted over all bands in the electromagnetic spectrum; to

\footnotetext{
1 Department of Physics and Astronomy, University of Leicester, Leicester LE1 7RH, UK; kpa@star.le.ac.uk.

2 Department of Physics and Astronomy, University of Nevada, Las Vegas, NV 89154-4002.

3 NASA Goddard Space Flight Center, Greenbelt, MD 20771.

4 Astrophysics Research Institute, Liverpool John Moores University, Egerton Wharf, Birkenhead CH41 1LD, UK.

5 Denver Research Institute, University of Denver, Denver, CO 80208.

6 Visiting Scholar, Stanford University.

7 Ioffe Physico-Technical Institute, Laboratory for Experimental Astrophysics, Saint Petersburg 194021, Russian Federation.

8 Istituto Nazionale di Astrofisica, Osservatorio Astronomico di Brera, I-23807 Merate (LC), Italy.

9 Dipartimento di Fisica, Universitá di Milano-Bicocca, I-20126 Milano, Italy.

10 Mullard Space Science Laboratory, University College London, Holmbury St. Mary, Dorking, Surrey RH5 6NT, UK.

11 University of Michigan, Ann Arbor, MI 48104.

12 INAF-IASF, Sezione di Palermo, 90146 Palermo, Italy.

13 Department of Astronomy and Astrophysics, Pennsylvania State University, University Park, PA 16802.

14 XMM-Newton Science Operations Centre, European Space Agency, Villafranca del Castillo, E-28080 Madrid, Spain.

${ }^{15}$ Center for Space Research, Massachusetts Institute of Technology, Cambridge, MA 02139.

16 Department of Physics and Astronomy, Louisiana State University, Baton Rouge, LA 70803.
}

understand GRBs as fully as possible, panchromatic observations are required over all time frames of the burst.

The Swift multiwavelength observatory (Gehrels et al. 2004) is designed to detect and follow up GRBs. With its rapid slewing ability, Swift is able to follow bursts and their afterglows from less than a minute after the initial trigger and can often still detect them weeks, and sometimes months, later. On rare occasions, such as when Swift triggers on a precursor to the main burst, the prompt emission, as well as the afterglow, can be observed at X-ray and UV/optical wavelengths. GRB 061121, the subject of this paper, is only the third GRB Swift has detected in this manner (after GRB 050117 and GRB 060124; Hill et al. 2006 and Romano et al. 2006, respectively), out of the almost 200 bursts triggered on in the first 2 years of the mission. ${ }^{17}$ Of these, GRB 061121 is the second well-sampled event (GRB 060124 was the first), and the first for which the UV/Optical Telescope (UVOT) was in event mode.

In addition to the small number of precursor triggers, around $10 \%$ of Swift bursts show detectable emission over the Burst Alert Telescope (BAT) bandpass by the time the narrow field instruments (NFIs) are on target.

Besides the Swift observations of prompt emission, there have been a small number of prompt optical measurements of GRBs, thanks to the increasing number of robotic telescopes around the world. A variety of behaviors have been found, with some optical

17 GRB 050820A would possibly have also been in this category, but Swift entered the South Atlantic Anomaly (SAA) just as a dramatic increase in count rate began (Cenko et al. 2006; Page et al. 2005a, 2005b; Cummings et al. 2005; Chester et al. 2005); Swift does not actively collect data during SAA passages. 
(and infrared) light curves tracking the gamma-ray emission (e.g., GRB 041219A; Vestrand et al. 2005; Blake et al. 2005), while others appear uncorrelated (e.g., GRB 990123, Akerlof et al. 1999; Panaitescu \& Kumar 2007; although see also Tang \& Zhang 2006; GRB 050904, Boër et al. 2006; GRB 060111B, Klotz et al. 2006; GRB 060124, Romano et al. 2006). GRB 050820A (Vestrand et al. 2006) showed a mixture of both correlated and uncorrelated optical flux.

Where correlations exist between different energy bands, it is likely that there is a common origin for the components. In the uncorrelated cases, the optical emission may be due to an external reverse shock (e.g., Sari \& Piran 1999; Mészáros \& Rees 1999), while the prompt gamma rays are caused by internal shocks. Cenko et al. (2006) suggest that the early optical data for GRB 050820A are produced by the forward shock passing through the band. In the case of GRB 990123, Panaitescu \& Kumar (2007) have suggested that the gamma rays arose from inverse Comptonization, while the optical emission was due to synchrotron processes; they do not assume a specific mechanism for the energy dissipation, allowing for the possibility of either internal or reverse-external shocks.

It is unclear whether precursors are ubiquitous features of GRBs, often remaining undetected because of a low signal-tonoise ratio or being outside the energy bandpass of the detector, or whether only some bursts exhibit them. A detailed discussion of the precursor phenomenon is beyond the scope of this paper and will be addressed in a future publication.

In this paper we report on the multiwavelength observations of both the prompt and afterglow emission of GRB 061121. Section 2 details the observations made by Swift, Konus-Wind, XMM-Newton, ROTSE (Robotic Optical Transient Search Experiment), and the Faulkes Telescope North (FTN), with multiband comparisons being made. In $\S 3$, we discuss the precursor, prompt, and afterglow emission, with a summary given in $\S 4$.

Throughout the paper, the main burst $(\sim 60-200 \mathrm{~s}$ after the trigger) will be referred to as the prompt emission, and the emission seen over -5 to $+10 \mathrm{~s}$ as the precursor, where the BAT trigger time $T_{0}=0 \mathrm{~s}$. Errors are given at $90 \%$ confidence (e.g., $\Delta \chi^{2}=2.7$ for one interesting parameter) unless otherwise stated, and the convention $F_{\nu, t} \propto \nu^{-\beta} t^{-\alpha}$ (with the photon spectral in$\operatorname{dex} \Gamma=\beta+1$ where $d N / d E \propto E^{-\Gamma}$ ) has been followed. We have assumed a flat universe, with Hubble constant $H_{0}=70 \mathrm{~km} \mathrm{~s}^{-1}$ $\mathrm{Mpc}^{-1}$, cosmological constant $\Omega_{\Lambda}=0.73$, and $\Omega_{\text {matter }}=1-\Omega_{\Lambda}$.

\section{OBSERVATIONS AND ANALYSES}

Two years and one day after launch, the BAT (Barthelmy et al. 2005) triggered on a precursor to GRB 061121 at 15:22:29 UT on 2006 November 21 (Page et al. 2006b). Swift slewed immediately, resulting in the NFIs being on target and beginning to collect data $55 \mathrm{~s}$ (X-Ray Telescope [XRT]; Burrows et al. 2005b) and 62 s (UVOT; Roming et al. 2005) later. This enabled broadband observations of the main burst event, which peaked $\sim 75 \mathrm{~s}$ after the trigger, leading to spectacular multiwavelength coverage of the prompt emission. The most accurate Swift position for this burst was that determined by the UVOT: R.A. $=09^{\mathrm{h}} 48^{\mathrm{m}} 54.55^{\mathrm{s}}$, decl. $=-13^{\circ} 11^{\prime} 42.4^{\prime \prime}\left(\mathrm{J} 2000.0 ; 90 \%\right.$ confidence radius of $0.6^{\prime \prime}$; Marshall et al. 2006); the refined XRT position is only $0.1^{\prime \prime}$ from these coordinates (Page et al. 2006a).

GRB 061121 was declared a "burst of interest" by the Swift team (Gehrels et al. 2006a), to encourage an intensive groundand space-based follow-up program. In addition to the Swift observations, the prompt emission of GRB 061121 was detected by RHESSI (Bellm et al. 2006), Konus-Wind, and Konus-A
(Golenetskii et al. 2006). Later afterglow observations were obtained in the X-ray (XMM-Newton; Schartel 2006) and radio (Very Large Array [VLA]; ${ }^{18}$ Chandra \& Frail 2006) bands. The Australia Telescope Compact Array (ATCA) and Westerbork Synthesis Radio Telescope (WSRT) also observed in the radio band between $\sim 5.2$ and $\sim 6.2$ days after the burst but did not detect the afterglow (van der Horst et al. 2006a, 2006b), implying that it had faded since the VLA observation.

Likewise, extensive optical follow-up observations were performed: ROTSE-IIIa (Yost et al. 2006), FTN (Melandri et al. 2006), Kanata $1.5 \mathrm{~m}$ telescope (Uemura et al. 2006), the University of Miyazaki $30 \mathrm{~cm}$ telescope (Sonoda et al. 2006), MichiganDartmouth-MIT Observatory (MDM; Halpern et al. 2006a, 2006b; Halpern \& Armstrong 2006a, 2006b), Palomar 60 inch (P60; Cenko 2006), Automated Response Telescope (ART; Torii 2006), the Crimean Astrophysical Observatory (CrAO) $2.6 \mathrm{~m}$ telescope (Efimov et al. 2006a, 2006b), and SMARTS/ANDICAM (Small and Moderate Aperture Research Telescope System/A Novel Double-Imaging CAMera; at infrared wavelengths, too; Cobb 2006) all detected the optical afterglow. Spectroscopic observations were performed at the Keck telescope about 12 minutes after the trigger, finding a redshift of $z=1.314$ for the optical afterglow, based on absorption features (Perley \& Bloom 2006; Bloom et al. 2006).

GRB 061121 has the highest instantaneous peak flux of all the long bursts detected by Swift to date (e.g., L. Angelini et al. 2007, in preparation).

\subsection{Gamma-Ray Data}

2.1.1. BAT

2.1.1.1. Temporal Analysis

After the initial precursor, the BAT count rate returned to close to the instrumental background level, until $T_{0}+60 \mathrm{~s}$, at which point the much brighter main burst began. This is characterized by a series of overlapping peaks, each brighter than the previous one, after which the gamma-ray flux decayed (from $\sim T_{0}+75 \mathrm{~s}$ to $\sim T_{0}+140 \mathrm{~s}$ ). Event data were collected until almost $1 \mathrm{ks}$ after the trigger, thus covering the entire emission period.

$T_{90}$, over $15-150 \mathrm{keV}$, and incorporating both the precursor and main emission, is $81 \pm 5 \mathrm{~s}$, measured from 8.8 to $89.8 \mathrm{~s}$ after the trigger. ${ }^{19}$ Figure 1 shows the mask-weighted BAT light curve in the four standard energy bands $[15-25 \mathrm{keV}, 25-50 \mathrm{keV}$, $50-100 \mathrm{keV}, 100-150 \mathrm{keV} ; 64 \mathrm{~ms}$ binning between 50 and $80 \mathrm{~s}$ after the trigger, with $1 \mathrm{~s}$ bins at all other times; units of counts $\mathrm{s}^{-1}$

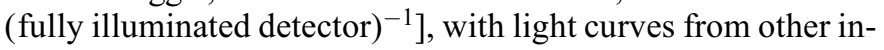
struments: the precursor and the pulses of the main burst are detected over all gamma-ray bands, although the precursor is only marginal over the 100-150 keV BAT band. There is also a soft tail (detected below $\sim 50 \mathrm{keV}$, when sufficiently coarse time bins are used) visible until about $140 \mathrm{~s}$ after the trigger (see bottom panel of Fig. 1), corresponding to a similar feature in the X-ray light curves.

\subsubsection{Spectral Analysis}

For the precursor, $T_{90, \text { pre }}=7.7 \pm 0.5 \mathrm{~s}(15-150 \mathrm{keV})$. A spectrum extracted over this interval can be well modeled by a single power law, with $\Gamma=1.68 \pm 0.09\left(\chi^{2} /\right.$ dof $\left.=26.2 / 23\right)$; no significant improvement was found by using the Band function

\footnotetext{
18 The National Radio Astronomy Observatory is a facility of the National Science Foundation operated under cooperative agreement by Associated Universities, Inc.

19 Errors on the BAT $T_{90}$ are estimated to be typically $5 \%-10 \%$, depending on the shape of the light curve.
} 

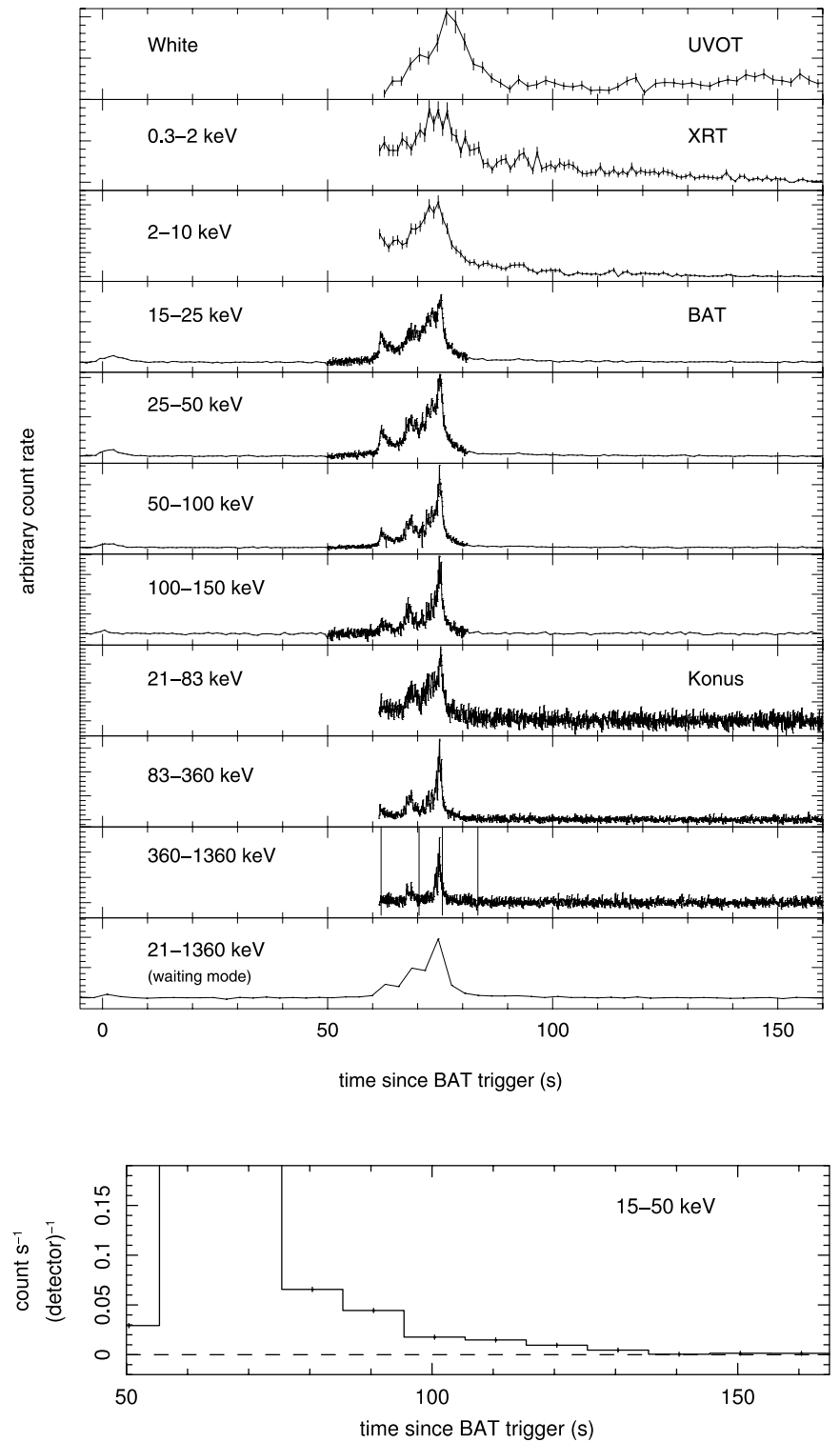

Fig. 1.-Top panels: Swift UVOT, XRT, BAT, and Konus-Wind light curves of GRB 061121; $1 \sigma$ error bars are shown for the UVOT and XRT data. Each instrument detected the peak of the main burst, with the precursor being detected over all gamma-ray energies. The vertical lines in the 360-1360 keV panel indicate the start and stop times for the spectra given in Table 1. Bottom panel: The $15-50 \mathrm{keV}$ BAT light curve, with $10 \mathrm{~s}$ bins, showing a tail out to $\sim 140 \mathrm{~s}$.

(Band et al. 1993) or a cutoff power law, and a thermal model led to a slightly $\left(\chi^{2} \sim 8\right)$ worse fit. The $15-150 \mathrm{keV}$ fluence for this time interval is $4 \times 10^{-7} \mathrm{ergs} \mathrm{cm}^{-2}$.

Considering only the main event, $T_{90, \text { main }}=18.2 \pm 1.1 \mathrm{~s}$ (measured from 61.8 to $80.0 \mathrm{~s}$ posttrigger). Fitting a power law to the mean spectrum during this time also results in a good fit $\left(\Gamma=1.40 \pm 0.01\right.$; fluence $=1.31 \times 10^{-5} \mathrm{ergs} \mathrm{cm}^{-2}$ over $15-$ $\left.150 \mathrm{keV} ; \chi^{2} / \mathrm{dof}=51.6 / 56\right)$; again, neither the Band function nor a cutoff power law improves on this. There is significant spectral evolution during the $T_{90}$ period, as shown in Figure 2: at times when the count rate is higher, the spectrum is harder. This behavior was also common in earlier bursts, as well as previous Swift detections (e.g., Golenetskii et al. 1983; Ford et al. 1995; Borgonovo \& Ryde 2001; Goad et al. 2007). The precursor shows a similar dependence of hardness ratio on count rate, suggesting that the emission processes in the precursor and the main burst are the same or similar.

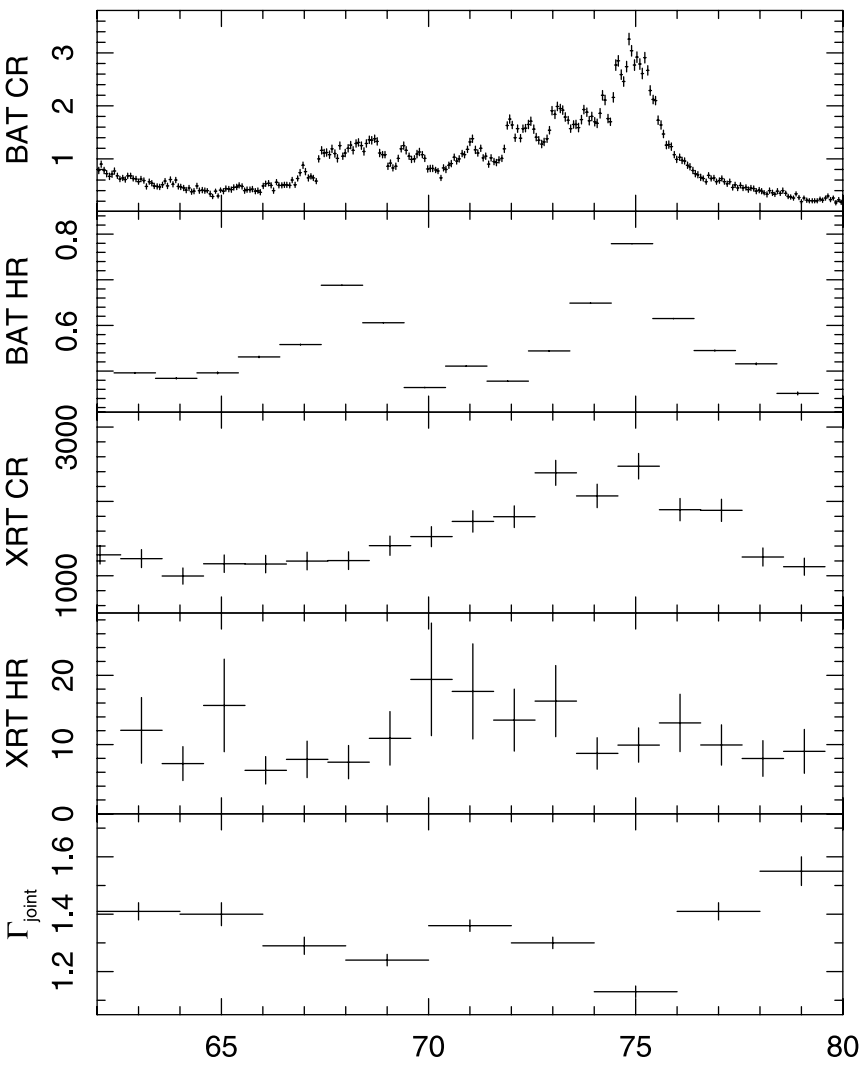

time since trigger (s)

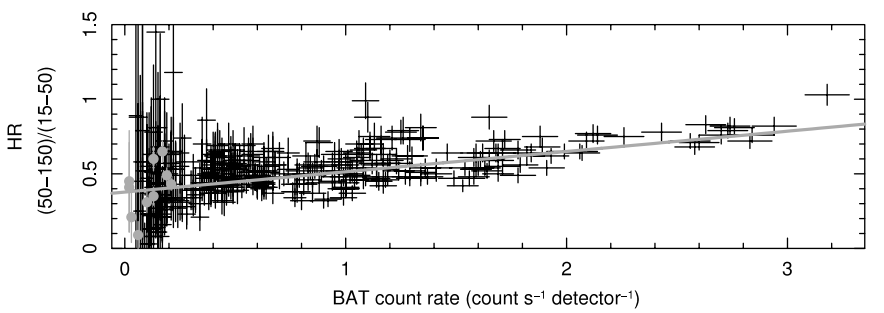

FIG. 2.-Top panels: Light curves, hardness ratios (HR), and the variation in $\Gamma$ using a single power-law fit during the main emission. The BAT light curve (top panel) is in units of counts $\mathrm{s}^{-1}$ (fully illuminated detector) ${ }^{-1}$, and the corresponding hardness ratio plots $(50-150 \mathrm{keV}) /(15-50 \mathrm{keV})$ using $1 \mathrm{~s}$ binning. The XRT light curve shows counts over $0.3-10 \mathrm{keV}$, while the hardness ratio compares $(1-10 \mathrm{keV}) /(0.3-1 \mathrm{keV})$ over $1 \mathrm{~s}$ bins. Bottom panel: BAT hardness ratio vs. count rate, showing that the emission is harder when brighter. Data from the precursor are shown as gray circles, with the main burst in black. The gray line shows a fit to the data, of the form $\mathrm{HR}=0.14 \mathrm{CR}+0.39$.

\subsubsection{Konus-Wind}

2.1.2.1. Temporal Analysis

Konus-Wind (Aptekar et al. 1995) triggered on the main episode of GRB 061121, while Konus-A triggered on the precursor (Golenetskii et al. 2006). Because of the spatial separation of Swift and Wind, the light-travel time between the spacecraft is $1.562 \mathrm{~s}$ : the Konus-Wind trigger time is $T_{0, \mathrm{~K}-W}=T_{0, \mathrm{BAT}}+$ $61.876 \mathrm{~s}$. All Konus light curves have been plotted with respect to the BAT trigger, corrected for the light-travel time. Figure 1 shows the Konus-Wind data plotted over the standard energy bands, with $64 \mathrm{~ms}$ binning; the bottom panel plots the coarser time resolution (2.944 s) "waiting mode" data, showing that Konus-Wind did see slightly enhanced emission at the time of the precursor. The background levels (which have been subtracted 
TABLE 1

Konus- $W$ ind Cutoff Power-Law Spectral Fit Results

\begin{tabular}{|c|c|c|c|c|c|}
\hline $\begin{array}{c}\text { Start Time } \\
\text { (s) }\end{array}$ & $\begin{array}{l}\text { Stop Time } \\
\text { (s) }\end{array}$ & $\Gamma$ & $\begin{array}{c}E_{\text {peak }} \\
(\mathrm{keV})\end{array}$ & $\Gamma_{\text {Band }}$ & $\chi^{2 / \text { dof }}$ \\
\hline 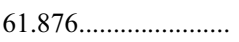 & 70.324 & $1.40_{-0.09}^{+0.08}$ & $478_{-99}^{+158}$ & $<2.1$ & $72 / 75$ \\
\hline 70.324...................... & 75.188 & $1.23_{-0.06}^{+0.05}$ & $608_{-71}^{+87}$ & $<2.9$ & $88 / 75$ \\
\hline $75.188 \ldots$ & 83.380 & $1.30_{-0.13}^{+0.11}$ & $621_{-159}^{+282}$ & $<2.3$ & $81 / 75$ \\
\hline $61.876 \ldots \ldots$ & 83.380 & $1.32_{-0.05}^{+0.04}$ & $606_{-72}^{+90}$ & $<2.7$ & $95 / 75$ \\
\hline
\end{tabular}

Notes.-Times are given with respect to the BAT trigger. $\Gamma_{\text {Band }}$ is the upper limit obtained for the spectral index above $E_{\text {peak }}$ when fitting with the Band function.

in each case) were 1005,370 , and 193.4 counts $\mathrm{s}^{-1}$ for bands $21-83 \mathrm{keV}, 83-360 \mathrm{keV}$, and $360-1360 \mathrm{keV}$, respectively.

\subsubsection{Spectral Analysis}

Table 1 gives the spectral fits to the Konus-Wind data in three separate time intervals shown by vertical lines in Figure 1 (KonusWind spectral intervals are automatically selected on board): up to the end of the "bump" around $70 \mathrm{~s}$ (the "start" of the burst), the burst maximum, and, finally, until most of the emission has died away (the burst tail). The data were fitted with a cutoff power law,

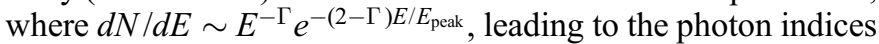
and peak energies given in the table. The Band function was used to estimate upper limits for the photon index above the peak; the values for the peak energy and $\Gamma$ obtained from the Band function were the same as when fitting the cutoff power law. Little variation in the spectral slope for energies below the peak is seen over these intervals, although the peak itself may have moved to somewhat higher energies during the burst emission. Extracting BAT spectra over the same time intervals and fitting with the same model (fixing $E_{\text {peak }}$ at the value determined from the KonusWind data) results in consistent spectral indices.

\subsection{X-Ray Data}

2.2.1. $X R T$

\subsubsection{Temporal Analysis}

The XRT identified and centroided on an uncataloged X-ray source in a $2.5 \mathrm{~s}$ Image Mode (IM) frame, as soon as the instrument was on target. This was quickly followed by a pseudo Piled-up Photo Diode (PuPD) mode frame. Following damage from a micrometeoroid impact in 2005 May (Abbey et al. 2005), the Photo Diode mode (Low Rate and Piled-up) has been disabled (for details on the different XRT modes see Hill et al. 2004); however, the XRT team is currently working on a method to reimplement these science modes and to update the ground software to process the files. The pseudo PuPD point presented here is the first use of such data.

Data were then collected in Windowed Timing (WT) mode starting at a count rate of $\sim 1280$ counts $\mathrm{s}^{-1}$ (pileup corrected; see below); the rate rapidly increased to a maximum of $\sim 2500$ counts s $^{-1}$ at $T_{0}+75 \mathrm{~s}$, making GRB 061121 the brightest burst yet detected by the XRT. Following this peak, the count rate decreased, with a number of small flares superimposed on the underlying decay (see Fig. 1). Photon Counting (PC) mode was automatically selected when the count rate was below about 10 counts $\mathrm{s}^{-1}$. Around $1.5 \mathrm{ks}$, the XRT switched back into WT mode briefly, due to an enhanced background linked to the sunlit Earth and a relatively high CCD temperature.

Because of the high count rate, the early WT data were heavily piled up; see Romano et al. (2006) for information about pileup in this mode. To account for this, an extraction region was used

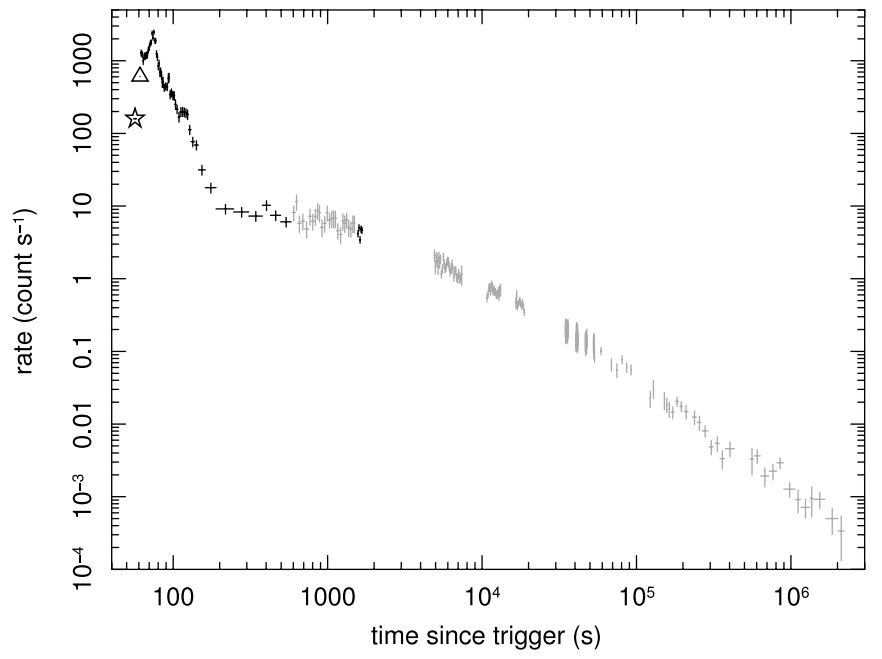

FIG. 3.-Swift XRT light curve of GRB 061121. The star and triangle show the initial Image Mode and pseudo-PuPD point (see text for details), followed by WT mode data (black) during the main burst (and at the end of the first orbit) and PC mode data (gray).

that excluded the central 20 pixels (diameter; 1 pixel $=2.36^{\prime \prime}$ ) and extended out to a total width of 60 pixels. Likewise, the first three orbits of PC data were piled up, and the data were thus extracted using annular regions (inner exclusion diameter decreasing from 12 to 6 to 4 pixels as the afterglow faded; outer diameter 60 pixels). The count rate was then corrected for the excluded photons by a comparison of the ancillary response files (ARFs) generated with and without a correction for the point-spread function (PSF); the ratio of these files provides an estimate of the correction factor. Nousek et al. (2006) give more details on this method. Occasionally, the afterglow was partially positioned over the CCD columns disabled by micrometeoroid damage mentioned above. In these cases, the data were corrected using an exposure map.

From $T_{0}+3 \times 10^{5} \mathrm{~s}$ onward, the afterglow had faded sufficiently for a nearby (41.5" away), constant (count rate $\sim 0.003$ counts s ${ }^{-1}$ ) source to contaminate the GRB region; this source is coincident with a faint object in the Digitized Sky Survey and is marginally detected in the UVOT $V$ filter. Thus, beyond this time, the extraction region was decreased to a diameter of 30 pixels, and the count rates corrected for the loss in PSF (a factor of $\sim 1.08$ ). The spectrum of this nearby source can be modeled with a single power law of $\Gamma=1.5_{-0.1}^{+0.2}$, with $N_{\mathrm{H}}=\left(1.8_{-1.2}^{+1.6}\right) \times 10^{21} \mathrm{~cm}^{-2}$, in comparison with the Galactic value in this direction of $5.09 \times$ $10^{20} \mathrm{~cm}^{-2}$ (Dickey \& Lockman 1990).

Figure 3 shows the XRT light curve, starting with the IM point (for details on how IM data are converted to a count rate see Hill et al. 2006) and followed by the pseudo-PuPD mode data. The importance of these early pre-WT data is clear, confirming that the XRT caught the rise of the main burst.

After the bright burst, the afterglow began to follow the "canonical" decay, seen in many Swift bursts (Nousek et al. 2006; Zhang et al. 2006). Such a decay can be parameterized by a series of power-law segments; in this case, fitting the data beyond $200 \mathrm{~s}$ after the trigger ( $=125 \mathrm{~s}$ after the main peak), two breaks in the light curve were identified, with the decay starting off very flat ( $\alpha=0.38 \pm 0.08)$ and eventually steepening to $\alpha=$ $1.07_{-0.06}^{+0.04}$ at $\sim 2.3 \mathrm{ks}$ and then $\alpha=1.53_{-0.04}^{+0.09}$ at $\sim 32 \mathrm{ks}$ (Table 2). The addition of the second break vastly improved the fit by $\Delta \chi^{2}=112.4$ for 2 degrees of freedom. However, we note that O'Brien et al. (2006) and Willingale et al. (2007) advocate a 
TABLE 2

XRT Power-Law Light-Curve Fits

\begin{tabular}{|c|c|c|}
\hline Parameter & Value & Epoch \\
\hline$\alpha_{1} \ldots \ldots \ldots \ldots$ & $0.38 \pm 0.08$ & Plateau phase \\
\hline$T_{\text {break }, 1}(\mathrm{~s}) \ldots \ldots \ldots \ldots$ & $2258_{-377}^{+507}$ & Plateau phase \\
\hline$\alpha_{2} \ldots \ldots \ldots \ldots \ldots \ldots \ldots \ldots \ldots \ldots \ldots \ldots \ldots$ & $1.07_{-0.06}^{+0.04}$ & Shallow phase \\
\hline$T_{\text {break }, 2}(\mathrm{~s}) \ldots \ldots \ldots \ldots$ & $\left(3.2_{-0.6}^{+2.1}\right) \times 10^{4}$ & Shallow phase \\
\hline$\alpha_{3} \ldots \ldots \ldots \ldots \ldots \ldots \ldots \ldots \ldots \ldots \ldots \ldots$ & $1.53_{-0.04}^{+0.09}$ & Steep phase \\
\hline
\end{tabular}

Notes.-XRT power-law light-curve fits from $200 \mathrm{~s}$ after the trigger onward; times are referenced to the BAT trigger. The names used in the text for the different epochs of the light curve are listed in the last column.

different description of the temporal decline; we return to this in $\S 3$.

Fitting the decay of the main peak $\left(75-200 \mathrm{~s}\right.$, keeping $T_{0}$ as the trigger time) with a power law, the slope is very steep, with $\alpha_{0}=5.1 \pm 0.2$. However, both Zhang et al. (2006) and Liang et al. (2006) have shown that the appropriate time origin is the start of the last pulse. Thus, a model of the form $f(t) \propto\left(t-t_{0}\right)^{-\alpha_{0}}$ was used, finding $t_{0}=58 \pm 1 \mathrm{~s}$ and a slope of $\alpha_{0}=2.2_{-0.3}^{+0.4}$; this is a statistically significant improvement on the power-law fit using the precursor $T_{0}\left(\Delta \chi^{2}=32\right.$ for one extra parameter).

Figure 4 plots the Swift data in terms of flux (the BAT data have been extrapolated into the $0.3-10 \mathrm{keV}$ band, using the joint fits with the XRT described in $\S 2.4 .1$ ) and flux density for UVOT. The BAT and XRT data are fully consistent with each other at all overlapping times.

\subsubsection{Spectral Analysis}

The XRT data also show that strong spectral evolution was present throughout the period of the prompt emission; this is discussed in conjunction with the BAT data in $\S$ 2.4.1. Considering the X-ray data alone, there is some indication that the spectra may be better modeled with a broken, rather than single, power law, although the break energies cannot always be well constrained (see Fig. 5). For each spectrum (covering periods of $2 \mathrm{~s}$ during the main pulse, followed by two spectra of $5 \mathrm{~s}[80-85 \mathrm{~s}$ and $85-90 \mathrm{~s}$ ] where the emission is fainter), the low-energy

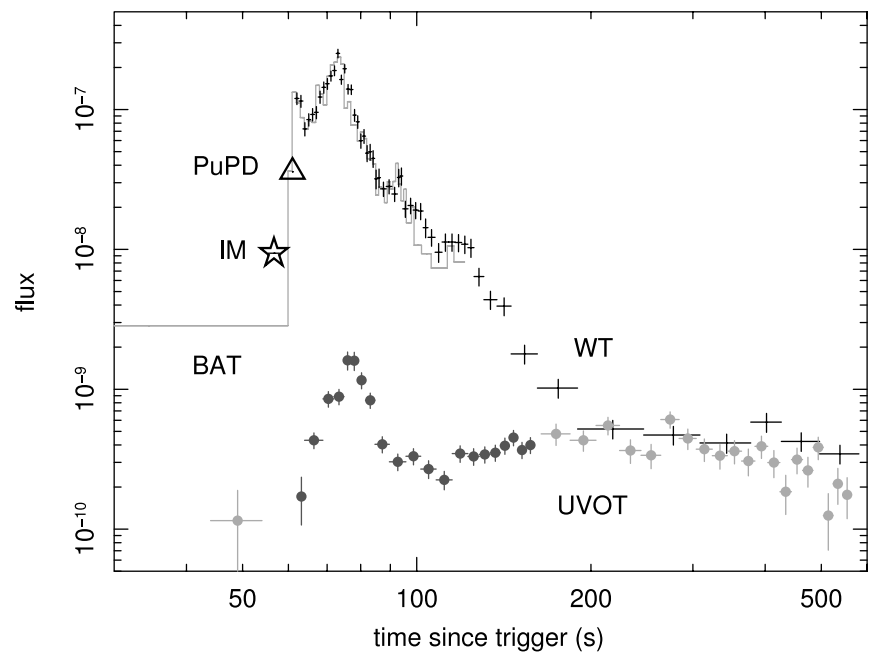

FIG. 4.- Swift flux light curve of GRB 061121, showing the early X-ray data (star, triangle, and crosses) and the BAT data (gray histogram) extrapolated into the $0.3-10 \mathrm{keV}$ bandpass in units of ergs $\mathrm{cm}^{-2} \mathrm{~s}^{-1}$, together with the UVOT flux density light curve (light gray circles: $V$ band; dark gray circles: White filter) in units of ergs cm $\mathrm{cm}^{-2} \mathrm{~s}^{-1} \AA^{-1}$, scaled to match the XRT flux observed at the start of the plateau phase.

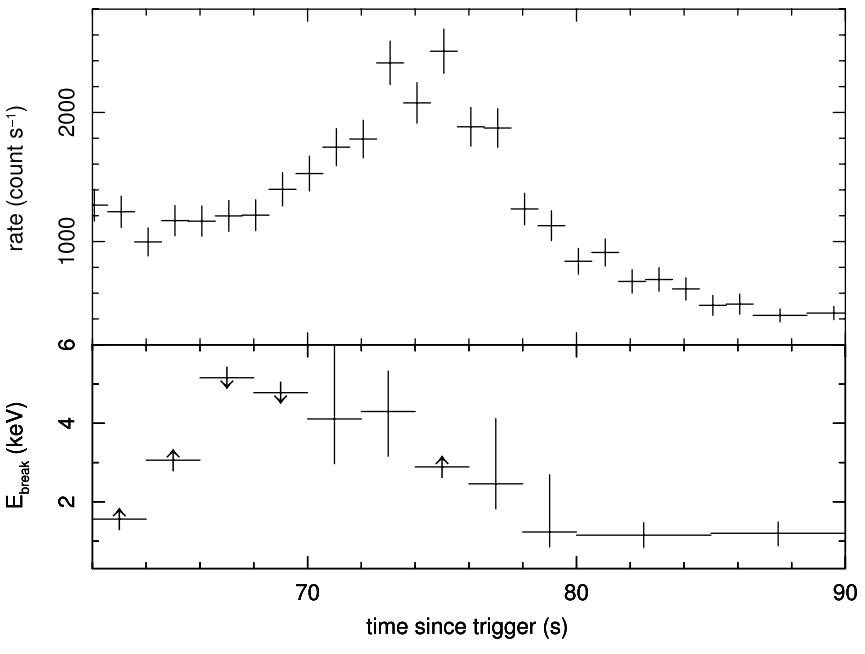

FIG. 5.- Fitting the X-ray data over $0.3-10 \mathrm{keV}$ with a broken power law $\left(\Gamma_{1}=0.69_{-0.07}^{+0.13}\right.$ and $\Gamma_{2}=1.61_{-0.13}^{+0.14}$ for all spectra), the break energy seems to move through the band, toward higher energies when the emission is brighter. Arrows indicate upper or lower $90 \%$ limits.

slopes were tied together for each spectrum (i.e., the slope measured is that averaged over all of the spectra), as were the highenergy indices, and the rest-frame column density, $N_{\mathrm{H}, z}$, was fixed at $(9.2 \pm 1.2) \times 10^{21} \mathrm{~cm}^{-2}$ from the best fit to the data from later times (see below); only the break energy and the normalization were allowed to vary. When simultaneously fitting all 11 spectra, $\chi^{2} /$ dof decreased from $142 / 134$ to $127 / 132$. Individually, the spectral fits were typically improved by $\chi^{2}$ of between 2 and 5.

The X-ray data during the GRB 051117A flares (Goad et al. 2007) were found to be better modeled with broken power laws, with the break energy moving to harder energies during each flare rise, and then softening again as the flux decayed. Likewise, Guetta et al. (2007) found breaks in the X-ray spectra obtained during the flares in GRB 050713A. The same pattern may be occurring here, and there is certainly an indication of spectral curvature.

The observed flux calculated from the spectrum corresponding to the peak of the emission (74-76 s) was measured to be $1.66 \times 10^{-7}$ ergs cm ${ }^{-2} \mathrm{~s}^{-1}$ (over $0.3-10 \mathrm{keV}$ ); the unabsorbed value was $1.77 \times 10^{-7} \mathrm{ergs} \mathrm{cm}^{-2} \mathrm{~s}^{-1}$.

The PC spectra were also extracted for the various phases of the light curve ("plateau," "shallow," and "steep;" defined in Table 2); the results of the fitting are presented in Table 3. In each phase, the spectrum could be well modeled by a single power law (no break required), with excess absorption in the rest frame of the GRB (modeled using ZTBABS and the "Wilms" abundance in XSPEC; Wilms et al. 2000). Together with the WT spectrum from $\sim 200$ to $590 \mathrm{~s}$ after the trigger (in the plateau stage), the first two PC spectra (plateau and shallow) are fully consistent with a constant photon index of $\Gamma=2.07 \pm 0.06$ and $N_{\mathrm{H}, z}=(9.2 \pm 1.2) \times 10^{21} \mathrm{~cm}^{-2}$.

Following the second apparent break in the light curve, around $3.2 \times 10^{4} \mathrm{~s}$, the spectrum hardened slightly, to a photon index of $\Gamma=1.83 \pm 0.11$ (or $1.87 \pm 0.08$ using $N_{\mathrm{H}, z}=9.2 \times 10^{21} \mathrm{~cm}^{-2}$ ).

\subsubsection{XMM-Newton}

XMM-Newton (Jansen et al. 2001) performed a Target of Opportunity observation of GRB 061121 (observation ID 0311792101) less than $6.5 \mathrm{hr}$ after the trigger (Schartel 2006) and collected data for $\sim 38 \mathrm{ks}$ (MOS1, MOS2; Turner et al. 2001) and $\sim 35 \mathrm{ks}$ ( pn; Strüder et al. 2001). This observation is mainly during the 
TABLE 3

XRT PC SPECTRAL Fits

\begin{tabular}{|c|c|c|c|c|c|}
\hline$\Gamma$ & $\begin{array}{c}N_{\mathrm{H}, z} \\
\left(10^{21} \mathrm{~cm}^{-2}\right)\end{array}$ & $\chi^{2} / \nu$ & Corresponding $\alpha$ & Epoch & $\begin{array}{c}\text { Time Since Trigger } \\
(\mathrm{s})\end{array}$ \\
\hline $2.14 \pm 0.12 \ldots \ldots \ldots \ldots$ & $10.8_{-2.8}^{+2.5}$ & $62.5 / 52$ & $0.38 \pm 0.08$ & Plateau & $590-1560$ \\
\hline $2.04 \pm 0.10 \ldots \ldots \ldots \ldots \ldots$ & $8.9_{-2.4}^{+2.1}$ & $67.5 / 70$ & $1.07_{-0.06}^{+0.04}$ & Shallow & $4900-22245$ \\
\hline $1.83 \pm 0.11 \ldots \ldots \ldots \ldots \ldots$ & $8.0_{-2.2}^{+2.4}$ & $48.0 / 55$ & $1.53_{-0.04}^{+0.09}$ & Steep & $34550-1152750$ \\
\hline $2.09 \pm 0.08 \ldots \ldots \ldots \ldots$ & $9.2 \pm 1.2$ (tied) & $63.5 / 53$ & $0.38 \pm 0.08$ & Plateau & $590-1560$ \\
\hline $2.05 \pm 0.06 \ldots \ldots \ldots \ldots \ldots$ & 9.2 (tied) & $67.6 / 71$ & $1.07_{-0.06}^{+0.04}$ & Shallow & $4900-22245$ \\
\hline $1.87 \pm 0.08 \ldots \ldots \ldots \ldots$ & 9.2 (tied) & $48.7 / 56$ & $1.53_{-0.04}^{+0.09}$ & Steep & $34550-1152750$ \\
\hline
\end{tabular}

NotEs.-XRT PC spectral fits, with rest-frame $N_{\mathrm{H}}$ free and then tied between all three spectra. The temporal decay slopes, $\alpha$, corresponding to each stage are also given. The Galactic absorbing column of $N_{\mathrm{H}}=5.09 \times 10^{20} \mathrm{~cm}^{-2}$ was always included in the model.

shallow phase, although it also covers a short time span after the break at around $32 \mathrm{ks}$.

Figure 6 plots the pn flux light curve and hardness ratio during the $X M M-N e w t o n$ observation, showing the lack of spectral evolution during this time frame; a hardness ratio calculated for the Swift data was in agreement with this finding. The decay slope over this time (MOS1, MOS2, pn, and joint) is consistent with the Swift results ( $\alpha \sim 1.3$; note that this crosses the time of the second break in the decay).

The XMM-Newton European Photon Imaging Camera (EPIC) spectra show clear evidence for excess $N_{\mathrm{H}}$, in agreement with the Swift data. In addition, fitting with excess $N_{\mathrm{H}}$ in the rest frame of the GRB gives a significantly better fit than at $z=0$, as shown in Figure 7. When fitting in the observer's frame, there is a noticeable bump in the residuals around $0.6 \mathrm{keV}$; fitting with $N_{\mathrm{H}}$ at $z=1.314$ removes this feature. The data are of sufficiently high signal-to-noise ratio that the redshift of the absorber can be estimated from the spectrum. Limits can be placed on the redshift and absorbing column, respectively, of $z>1.2$ and $N_{\mathrm{H}, z}>4.6 \times$ $10^{21} \mathrm{~cm}^{-2}$ at $99 \%$ confidence, in agreement with the spectroscopic redshift from Bloom et al. (2006) within the statistical uncertainties. At their value of $z=1.314$, the excess $N_{\mathrm{H}_{z} z}$ from the EPIC pn spectrum is $(5.3 \pm 0.2) \times 10^{21} \mathrm{~cm}^{-2}$, lower than the best fit to the Swift data from the simultaneous shallow decay section, but more similar to the values obtained from fitting the optical to X-ray spectral energy distributions (SEDs) in $\S 2.4 .2$. In agreement with the simultaneous XRT PC mode data, there is

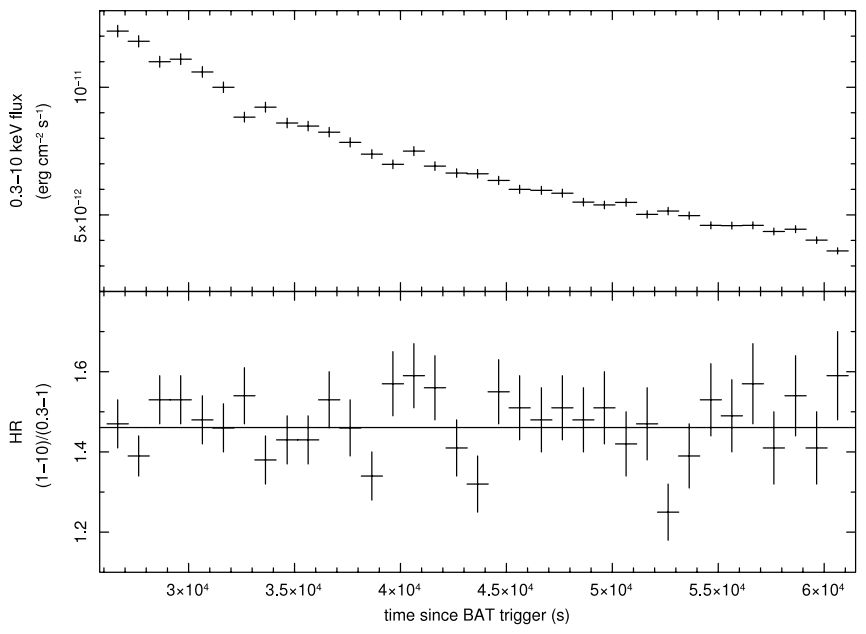

FIG. 6.-XMM-Newton EPIC pn light curve and hardness ratio of GRB 061121. The horizontal line shows that the hardness ratio is consistent with a constant value of $\sim 1.46$, indicating that there is no spectral evolution during this time. no evidence for a break in the EPIC spectrum over this time period. Spectra from neither the Reflection Grating Spectrometer (den Herder et al. 2001) nor EPIC show obvious absorption or emission lines.

\subsubsection{Chandra}

Chandra performed a $33 \mathrm{ks}$ Target of Opportunity observation at $\sim 61$ days after the trigger. No source was detected at the position of the X-ray afterglow, with a $3 \sigma$ upper limit of $2.5 \times 10^{-15} \mathrm{ergs} \mathrm{cm}^{-2} \mathrm{~s}^{-1}$.

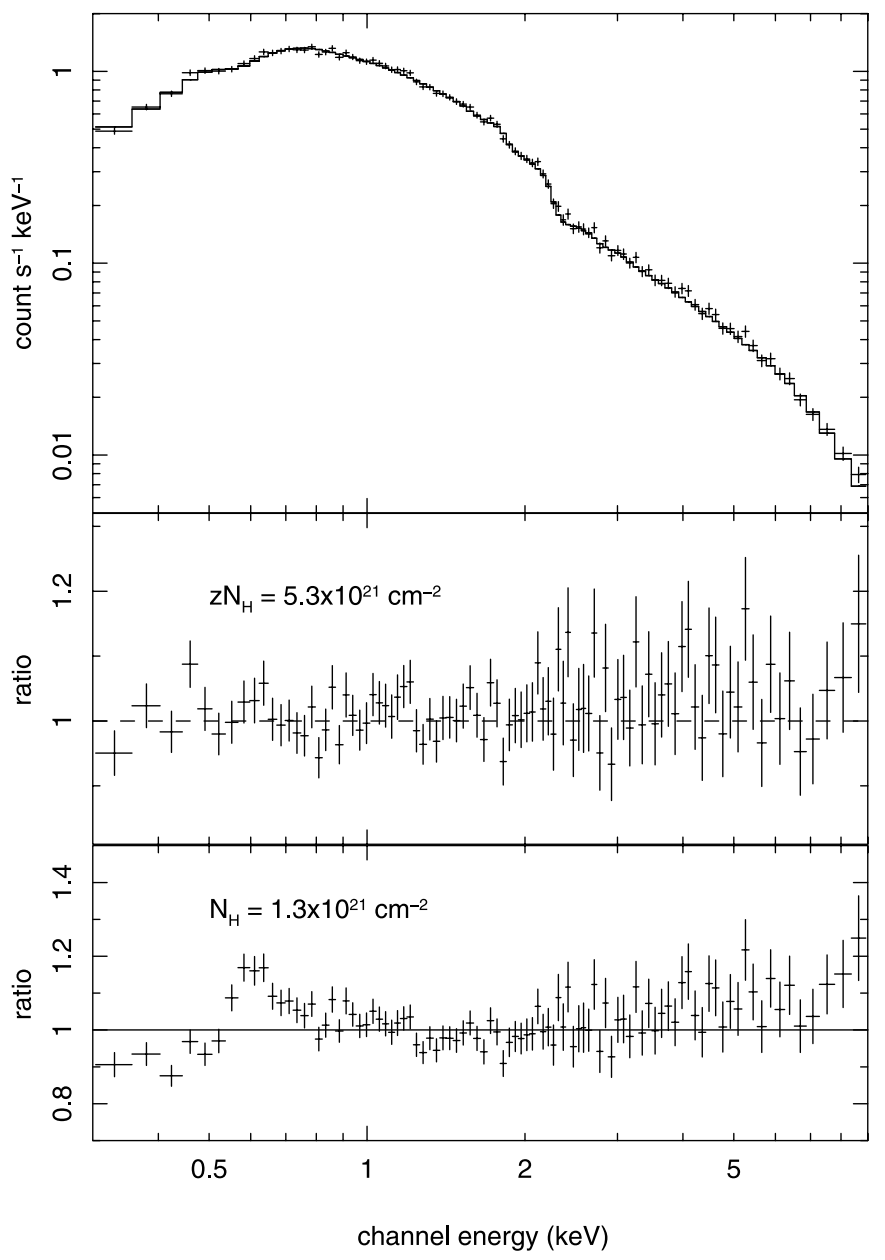

FIG. 7.-EPIC pn spectrum of the late-time afterglow of GRB 061121, with an excess absorbing column in both the rest frame of the GRB and the observer's frame. The spectrum is much better modeled with an excess column at $z=1.314$ 


\subsection{Optical/UV Data}

\subsubsection{UVOT}

The UVOT detected an optical counterpart in the initial White filter ${ }^{20}$ observation, starting $62 \mathrm{~s}$ after the trigger, and subsequently in all other filters (optical and UV). The UVOT followed the typical sequence for GRB observations, with the early data being collected in event mode, which has a frame time of $8.3 \mathrm{~ms}$ during this observation. ${ }^{21}$ Photometric measurements were obtained from the UVOT data using a circular source extraction region with a $5^{\prime \prime}-6$ " radius. UVOTMAGHIST was used to convert count rates to magnitudes and flux; no normalization between the different filters was applied.

As in the gamma-ray and X-ray bands, the main burst was detected, with an increase in count rate seen between $\sim 50$ and $75 \mathrm{~s}$ after the trigger (Figs. 1 and 4). However, although an increase in count rate is seen for the UVOT data, it is by a smaller factor than observed for the XRT. After $\sim 110 \mathrm{~s}$, the UVOT emission stops decaying and rebrightens slightly, until $140 \mathrm{~s}$ after the trigger, at which time it flattens off and then starts to fade again (Fig. 4). The slower decay between $\sim 100$ and 200 s may be indicative of the contribution of an additional (afterglow) component beginning to dominate.

A single UV/optical light curve was created from all the UVOT filters in order to get the best measurement of the optical temporal decay. This was done by fitting each filter data set individually (between 200 and $1 \times 10^{5} \mathrm{~s}$ ) and finding the normalization, which was then modified to correspond to that of the $V$-band light curve. The decay across all the filters beyond $200 \mathrm{~s}$ after the trigger can be fitted with a single slope of $\alpha_{\mathrm{UVOT}}=$ $0.68 \pm 0.02$; the individual $U, B$, and $V$ decay rates are consistent with one another. No break in the light curve is seen out to $\sim 100 \mathrm{ks}$.

\subsubsection{ROTSE}

ROTSE-IIIa, at the Siding Spring Observatory in Australia, first imaged GRB $06112121.6 \mathrm{~s}$ after the trigger time under poor (windy) seeing conditions. A variable source was immediately identified, at a position coincident with that determined by the UVOT (Yost et al. 2006).

The ROTSE data (unfiltered, but calibrated to the $R$ band) have been included in Figure 10 (discussed later). It is noticeable that the peak around 75 s seen in the Swift data is not readily apparent in the ROTSE measurements. The bandpass of the UVOT White filter is more sensitive to photons with wavelengths of $\lambda<4500 \AA$, ${ }^{22}$ while the ROTSE bandpass is redder. This, together with poor seeing conditions during the observation, may explain why the ROTSE light curve does not clearly show the main emission.

\subsubsection{Faulkes Telescope North}

The FTN, at Haleakala on Maui, Hawaii, began observations of GRB $061121225 \mathrm{~s}$ after the burst trigger, performing a $B V R i^{\prime}$ multicolor sequence (Melandri et al. 2006). $R$-band photometry was performed relative to the USNO-B1.0 "R2" magnitudes. Magnitudes were then corrected for Galactic extinction using the dust extinction maps by Schlegel et al. (1998) and converted to fluxes using the absolute flux calibration from Fukugita et al.

\footnotetext{
20 The White filter covers a broad bandpass of $\lambda \sim 1600-6500 \AA$.

21 The data have been adjusted to take into account an incorrect onboard setting (between 2006 November 10 and 22), which resulted in the wrong frame times being stored in the headers of the UVOT files (Marshall 2006).

${ }_{22}$ See http://swiftsc.gsfc.nasa.gov/docs/heasarc/caldb/swift/.
}

(1995). The photometric $R$-band points have been included in Figure 10.

\subsection{Broadband Modeling}

\subsubsection{Gamma Rays to X-Rays}

2.4.1.1. Spectral Analysis

Because the BAT was in event mode throughout the observation of the main burst of GRB 061121, detailed spectroscopy could be performed. Unfortunately, this was not the case during the prompt observation of GRB 060124 (Romano et al. 2006).

Figure 2 demonstrates the spectral evolution seen in both the BAT and XRT during the prompt emission. Spectra were extracted over $2 \mathrm{~s}$ intervals, in an attempt to obtain sufficient signal-to-noise ratio while not binning over too much of the rapid variability. The BAT data are hardest around 68 and $75 \mathrm{~s}$ (the second of these times corresponding to the peak of the main emission); the XRT hardness peaks at about $70 \mathrm{~s}$, which could be a further indication of the softer data lagging the harder. The joint spectrum $\left(\Gamma_{\text {joint }}\right.$ comes from a simple absorbed power-law fit to the simultaneous BAT and XRT data) is at its hardest during the brightest part of the emission. The joint fit also hardens around 68-70 s, between the times when the BAT and XRT data, respectively, are at their hardest. The onboard spectral time bin selection prevents the Konus-Wind data from being sliced into corresponding times, so constraints have not been placed on the high-energy cutoff, $E_{\text {peak }}$. Breaks in the XRT/BAT power laws can only be poorly constrained.

In Figure 4, the BAT and XRT data were converted to $0.3-$ $10 \mathrm{keV}$ fluxes using the time-sliced power-law fits to the simultaneous BAT and XRT spectra. Without the use of such varying conversion factors, the derived BAT and XRT fluxes would have been inconsistent with each other.

A broadband spectrum, covering $0.3 \mathrm{keV}$ to $4 \mathrm{MeV}$ in the observer's frame (XRT, BAT, and Konus-Wind) for $\sim 70-75 \mathrm{~s}$ post trigger, was fitted by the absorbed cutoff power-law model described in $\S 2.1 .2$. A constant factor of up to $10 \%$ was included between the BAT and Konus-Wind data, to allow for calibration uncertainties. The best fit $\left(\chi^{2} /\right.$ dof $\left.=301 / 167\right)$ gives $\Gamma=$ $1.19 \pm 0.01$, with $E_{\text {peak }}=670_{-47}^{+65} \mathrm{keV} . N_{\mathrm{H}, z}$ was fixed at $9.2 \times$ $10^{21} \mathrm{~cm}^{-2}$ (from the X-ray fits in $\S 2.2 .1$ ). Allowing $\Gamma$ to vary between the three spectra hints at further spectral curvature, although the differences are marginal, significant at only the $2 \sigma$ level.

The isotropic equivalent energy (calculated using the timeintegrated flux over the full $T_{90}$ period) is $2.8 \times 10^{53} \mathrm{ergs}$ in the $1 \mathrm{keV}-10 \mathrm{MeV}$ band (GRB rest frame), meaning that GRB 061121 is consistent with the Amati relationship (Amati et al. 2002). See $\S 3.3 .2$ for a beaming-corrected gamma-ray energy limit.

$$
\text { 2.4.1.2. Lag Analysis }
$$

A lag analysis (e.g., Norris et al. 1996) between the BAT bands leads to interesting results. Comparing bands $50-100 \mathrm{keV}$ and $15-25 \mathrm{keV}$, the precursor emission yields a spectral lag of $600 \pm 100 \mathrm{~ms}$, while the main emission has a much smaller lag of $1 \pm 6 \mathrm{~ms}$. Note that the calculation was performed using $64 \mathrm{~ms}$ binning for the precursor and $4 \mathrm{~ms}$ binning for the main burst; see Norris (2002) and Norris \& Bonnell (2006) for more details on the procedure. This lag for the main emission is rather small for a typical long burst; however, both lags are consistent with the long-burst luminosity-lag relationship generally seen (Norris et al. 2000). The short spectral lag for the main emission and the longer value for the precursor are also found when comparing the $100-350 \mathrm{keV}$ and $25-50 \mathrm{keV}$ bands. 


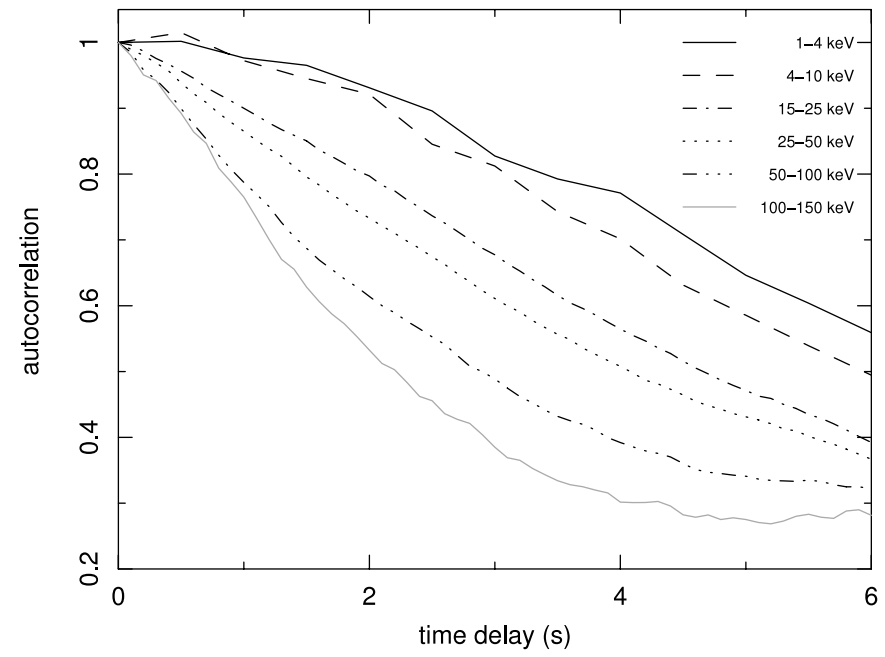

FIG. 8.- Autocorrelation function of the BAT and XRT data during the prompt emission of GRB 061121, showing that the main burst peak is broader at softer energies.

Similarly, comparison of the hard and soft $(2-10 \mathrm{keV}$ and $0.3-2 \mathrm{keV}$ ) XRT bands reveals a lag of approximately $2.5 \mathrm{~s}$, as the emission softens through the main burst. The X-ray data also lag behind the gamma-ray data, and the optical behind the X-ray.

Link et al. (1993) and Fenimore et al. (1995) used a sample of the Burst And Transient Source Experiment (BATSE; Paciesas et al. 1999) bursts to investigate the relationship between the duration of bursts and the energy band considered. They found that the bursts, as well as smaller structures within the main emission, generally become shorter with increasing energy (see also Cheng et al. 1995; Norris et al. 1996; in't Zand \& Fenimore 1996; Piro et al. 1998). Figure 8 plots the autocorrelation function over various X-ray and gamma-ray bands, to reinforce the point that the peak is narrower the harder the band, over X-ray as well as gammaray energies. Comparison of the light curves over the different energy bands in Figure 1 demonstrates this as well. A similar behavior was also found for GRB 060124, where Romano et al. (2006) compared the $T_{90}$ values obtained for the main burst over the X-ray and gamma-ray bands. Fenimore et al. (1995) found that the width of the autocorrelation function is $W \propto E^{-0.4}$, where $E$ is the energy at which the function was determined; the six measurements from GRB 061121 are consistent with this finding.

\subsubsection{Optical to X-Rays}

Using the Swift X-ray and UV/optical data, $R$ - and $i^{\prime}$-band data from the Faulkes Telescope, and $R_{\mathrm{C}}$ data from the Kanata telescope (Uemura et al. 2006), SEDs were produced at epochs corresponding to the peak of the emission ( $72-75 \mathrm{~s}$ post BAT trigger), the plateau stage, and during the shallow decay. Fitting at the different epochs gives an estimation of the broadband spectral variation.

For each of the UVOT lenticular filters, the tool UVOT2PHA was used to produce spectral files compatible with XSPEC, and for the latter two epochs the count rate in each band was set to that determined from a power-law fit to the individual filter light curves over the time interval in question, using $\alpha=0.68$. To determine the Faulkes Telescope $R$ - and $i^{\prime}$-band flux during the plateau stage, a power law was fitted to the complete data set (220-1229 s post BAT trigger for $R$ and $467-1401 \mathrm{~s}$ for $i^{\prime}$ ) with the decay index left as a free parameter. The $R$ magnitude at the midtime of the shallow stage $(6058 \mathrm{~s})$ was determined from the Kanata $R$-band magnitude reported at 6797 s (Uemura et al. 2006), assuming the same decay index as observed in the UVOT data. An uncertainty of 0.2 mag was assumed as the systematic uncertainty for the photometric calibration of the ground-based data.

At a redshift of $z=1.314$, the beginning of the Ly $\alpha$ forest is redshifted to an observer-frame wavelength of $\sim 2812 \AA$, which falls within the UVW1 filter bandpass, the reddest of the UV filters. A correction was applied to the three UV filter fluxes to account for this absorption, based on parameters from Madau (1995) and Madau et al. (1996); see also P. Curran et al. (2007, in preparation).

The methods used for simultaneous fitting of the SED components are described in detail in Schady et al. (2007). The SEDs were fitted with a power law, or a broken power law, as expected from the synchrotron emission, and two dust and gas components, to model the Galactic and host galaxy photoelectric absorption and dust extinction. The column density and reddening in the first absorption system were fixed at the Galactic values. [The Galactic extinction along this line of sight is $E(B-V)=$ 0.046 (Schlegel et al. 1998).]

The second photoelectric absorption system was set to the redshift of the GRB, and the neutral hydrogen column density in the host galaxy was determined assuming solar abundances. The dependence of dust extinction on wavelength in the GRB host galaxy was modeled using three extinction laws, taken from observations of the Milky Way (MW), the Large Magellanic Cloud (LMC), and the Small Magellanic Cloud (SMC) and parameterized by Pei (1992) and Cardelli et al. (1989). The greatest differences observed in these extinction laws are the amount of far-UV extinction (which is greatest in the SMC and least in the MW) and the strength of the $2175 \AA$ absorption feature (which is most prominent in the MW and negligible in the SMC).

Fitting these data together, a measurement of the spectral slope and optical and X-ray intrinsic extinctions (for the second two epochs) were obtained (Table 4); the $A_{V}$ values given in the table are in addition to the $A_{V}=0.151$ associated with the MW itself. The slope above the break energy (which lies toward the lowenergy end of the X-ray bandpass for each phase) was assumed to be exactly 0.5 steeper than the spectral slope below the break (the condition required for a cooling break), since allowing all of the parameters to vary leads to unconstrained fits. Figure 9 shows, as an example, the fit to the data in the plateau stage.

An MW dust extinction law provides the best overall fit to the data, using a broken power-law model, although the LMC model is equally acceptable.

During the plateau phase, and adopting the broken power-law model parameters given in Table 4 , we find gas-to-dust ratios of $(1.6 \pm 0.7) \times 10^{22},(2.6 \pm 0.7) \times 10^{22}$, and $(3.0 \pm 0.7) \times$ $10^{22} \mathrm{~cm}^{-2} \mathrm{mag}^{-1}$ for MW, LMC, and SMC fits, respectively. We can compare these estimates to the measured values for the MW of $(4.93 \pm 0.45) \times 10^{21} \mathrm{~cm}^{-2} \mathrm{mag}^{-1}$ (Diplas \& Savage 1994) and the LMC and SMC of $(2.0 \pm 0.8) \times 10^{22}$ and $(4.4 \pm 1.1) \times$ $10^{22} \mathrm{~cm}^{-2} \mathrm{mag}^{-1}$, respectively (Koornneef 1982; Bouchet et al. 1985). The MW fit to GRB 061121, which is found to be marginally the best model, is consistent with the LMC gas-to-dust ratio only, at the $90 \%$ confidence level. The ratios derived from the LMC and SMC fits are consistent with both the LMC and SMC gas-to-dust ratios. We note that all fits are inconsistent with the MW ratio at this confidence level, following the trend seen in pre-Swift bursts (e.g., Starling et al. 2007 and references therein), and that if a metallicity below solar were adopted, the gas-to-dust 
TABLE 4

Power-Law and Broken Power-Law Fits to the Simultaneous UVOT and XRT Spectra of GRB 061121

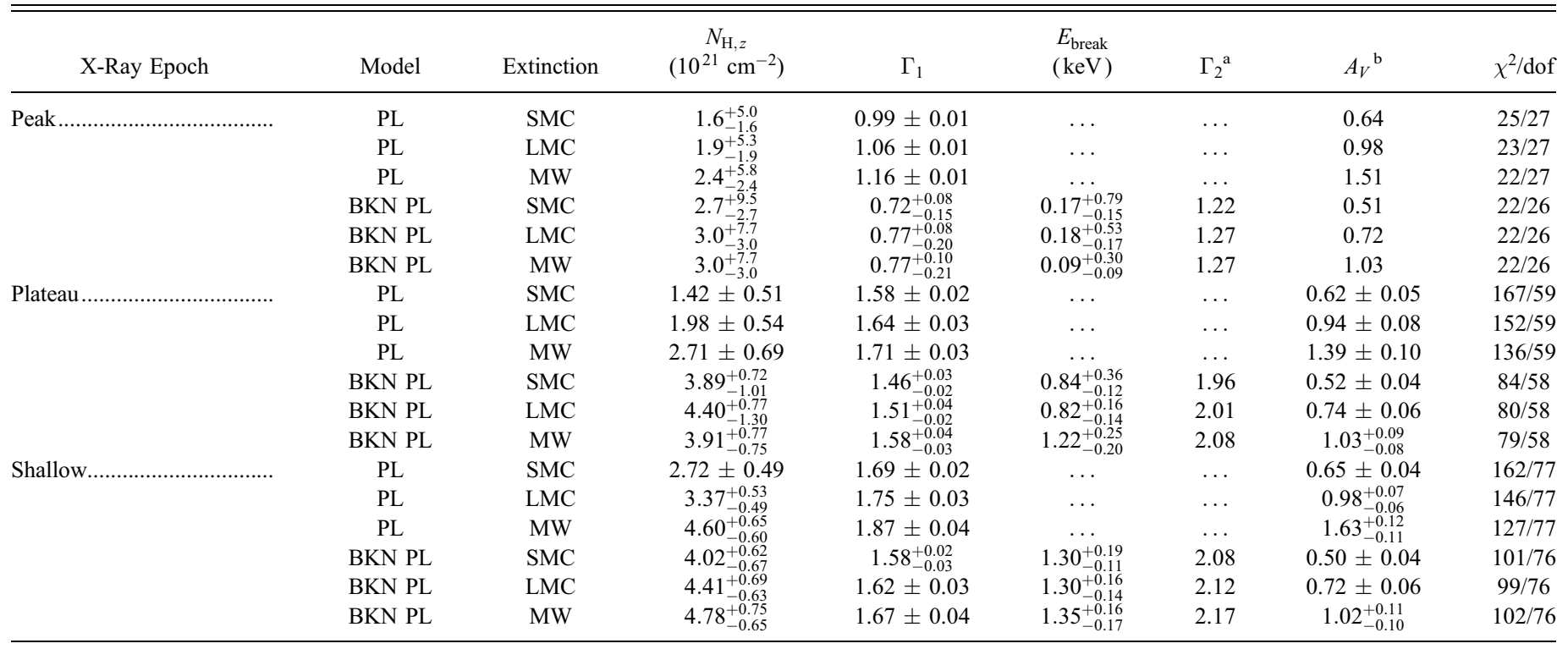

Notes.-Power-law (PL) and broken power-law (BKN PL) fits to the simultaneous UVOT and XRT spectra of GRB 061121, for three different dust extinction models: Small and Large Magellanic Clouds (SMC and LMC) and the Milky Way (MW). $\Gamma_{1}$ and $\Gamma_{2}$ are the photon indices below and above the spectral break for the BKN PL models, respectively. The data points have not been corrected for reddening.

${ }^{\text {a }} \Gamma_{2}$ is set to be equal to $\Gamma_{1}+0.5$ in each broken power-law fit, as would be expected if the change in index were due to a cooling break.

${ }^{\mathrm{b}}$ In the fit to the peak epoch, $A_{V}$ is fixed to the average best-fit value found in the same model fits to plateau and shallow stage data. The $A_{V}$ values are given for the observer's frame of reference.

ratio of GRB 061121 would increase, moving it further toward the $\mathrm{SMC}$ value.

\section{DISCUSSION}

Swift triggered on a precursor to GRB 061121 leading to comprehensive broadband observations of the prompt emission,

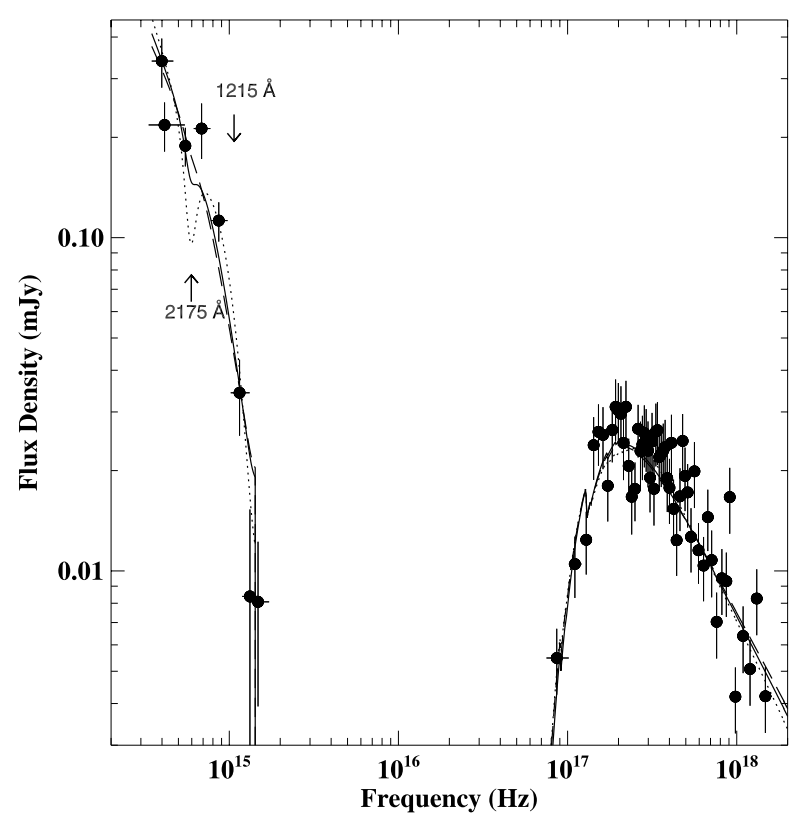

FIG. 9.- Broken power-law fit to the UVOT, XRT, and ground-based $R$ and $i^{\prime}$ SED of GRB 061121 between $\sim 596$ and $1566 \mathrm{~s}$ after the trigger (plateau phase) plotted in the observer's frame. The arrows indicate the beginning of the Ly $\alpha$ forest (1215 $\AA$ in the rest frame) and the absorption feature in the MW dust extinction law $(2175 \AA)$, which is shown by a dotted line. The solid line corresponds to the LMC extinction, and the dashed line to the SMC extinction. as well as the later afterglow. We discuss these here, together with possible mechanisms involved.

\subsection{Precursor}

Lazzati (2005) found that about $20 \%$ of BATSE bursts showed evidence for gamma-ray emission above the background between 10 and $\sim 200$ s before the main burst, typically with nonthermal spectra that tended to be softer than the main burst. GRB 060124 (Romano et al. 2006) and GRB 061121 show the same behavior.

Precursor models have been proposed for emission well separated from, or just prior to, the main burst. Early emission occurring only a few seconds before the main burst has been explained by the fireball interacting with the massive progenitor star, although the spectrum of such emission is expected to be thermal (Ramirez-Ruiz et al. 2002b). Lazzati et al. (2007) investigated shocks in a cocoon around the main burst; their model predicts a nonthermal precursor as the jet breaks out of the surface of the star. A high-pressure cocoon is formed as the subrelativistic jet head forces its way out of the star. As the head of the jet breaks through the surface, the energy of this cocoon is released through a nozzle and can give rise to a precursor (Ramirez-Ruiz et al. 2002a, 2002b). Within the framework of this model, observers located at viewing angles of $5^{\circ}<\theta<11^{\circ}$ are expected to see first a relatively bright precursor, then a dark phase with little emission, followed, when the jet enters the unshocked phase, by a bright GRB; this is very similar to the light curve observed for GRB 061121. Waxman \& Mészáros (2003) demonstrate that both a series of thermal X-ray precursors (becoming progressively shorter and harder) and nonthermal emission can be produced by an emerging shocked jet, although the nonthermal component is expected to be in the $\mathrm{MeV}$ range. There could also be an accompanying inverse Compton component, formed by the thermal $\mathrm{X}$-rays being upscattered by the jet. 
The same type of smooth, wide-pulse, low-intensity emission as seen in some precursors, but occurring after the main emission, is also occasionally seen (e.g., Hakkila \& Giblin 2004; Nakamura 2000). Hakkila \& Giblin (2004) discuss two examples where postcursor emission is found to have a longer lag than expected from the lag-luminosity relation, to have a smoother shape, and to be softer. In the case of the GRB 061121 precursor, the spectrum is, indeed, softer than the main event and shows a comparatively smooth profile. The emission does have a longer lag than the main emission, but it is still consistent with the lagluminosity relation.

There are two expected effects that could lead to such a difference in lags for separate parts of a single burst: the much lower luminosity for the precursor (resulting from a much smaller Lorentz factor; the measured fluence of the precursor is about a factor of 30 smaller than the fluence of the main emission) is a natural explanation, while the precursor being emitted at a greater offaxis angle could also have an effect. In this second case, ejecta are considered to emerge at different angles with respect to the jet axis; not all of the solid angle of the jet will be "filled" uniformly.

Such late postcursor emission is unlikely to be linked to the jet breakout from the stellar surface, and it may not be sensible to attribute apparently similar phenomena (in the form of pre- and postcursors) to entirely different processes.

Pre/postcursor emission could be due to the deceleration of a faster front shell, resulting in slower shells catching up and colliding with it (Fenimore \& Ramirez-Ruiz 1999; Umeda et al. 2005; note, however, that a faster shell would be inconsistent with the precursor having a smaller Lorentz factor as suggested to explain the lag discrepancy), or late activity of the central engine. The presence of flares in about $50 \%$ of Swift bursts is generally attributed to continuing activity of the central engine (Burrows et al. 2005a; Zhang et al. 2006), and the appearance of broken power laws in the X-ray spectra of both flares and the prompt emission (Guetta et al. 2007; Goad et al. 2007) hints of a common mechanism.

\subsection{Prompt Emission}

The prompt emission mechanism for GRBs is still debated and the origin of $E_{\text {peak }}$ is not fully understood (Mészáros et al. 1994; Pilla \& Loeb 1998; Lloyd \& Petrosian 2000; Zhang \& Mészáros 2002; Rees \& Mészáros 2005; Pe'er et al. 2005). The standard synchrotron model predicts fast cooling (Ghisellini et al. 2000) with a photon index, $\Gamma$, of $3 / 2$ and $(p / 2)+1$ below and above the peak energy, respectively (e.g., Zhang \& Mészáros 2004). The Konus-Wind spectral index below $E_{\text {peak }}$ is shallower than $3 / 2$, which may suggest a slow cooling spectrum with $p<2\left[E_{\text {peak }}\right.$ being the cooling frequency and $\Gamma=(p+1) / 2]$ or additional heating. A slow-cooling spectrum can be retained by assuming that the magnetic fields behind the shock decay significantly in $10^{4}-$ $10^{5} \mathrm{~cm}$, so that synchrotron emission happens in small-scale magnetic fields (Pe'er \& Zhang 2006).

The SED at the peak time (SED 2 in Fig. 11, discussed below) has a peak flux density of around $1 \mathrm{keV}$, below which the optical to X-ray spectral slope is $0.11 \pm 0.09$. This slope is harder than expected from the standard synchrotron model (which predicts an index of $\frac{1}{3}$ ). There should, however, be spectral curvature around the break, which could flatten the index (Lloyd \& Petrosian 2000), so the data could still be consistent with the synchrotron model. An alternative to synchrotron emission, in the form of "jitter" radiation, is discussed by Medvedev (2000), although that model predicts an even steeper index of 1 below the jitter break frequency.

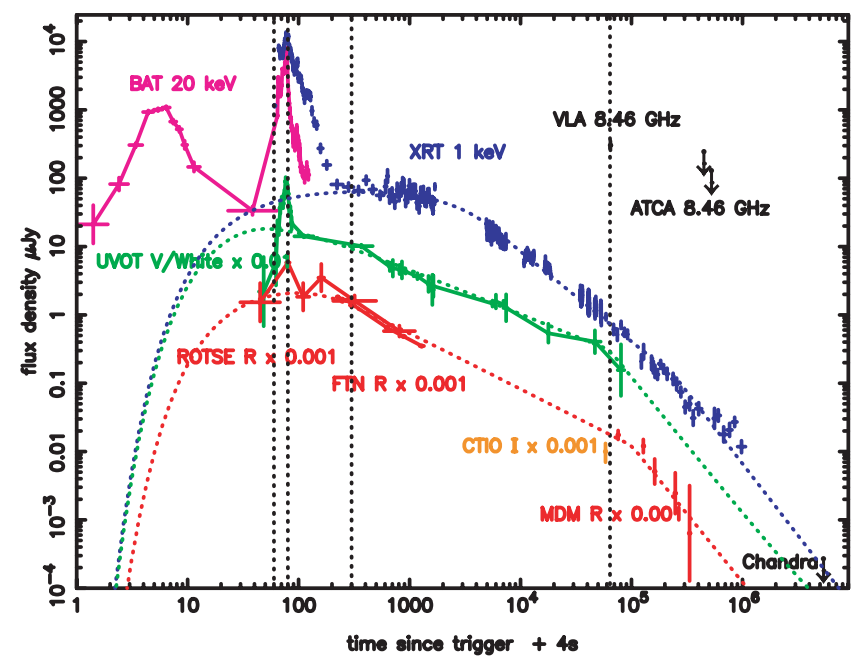

FIG. 10.-Flux density light curves for the gamma-ray, X-ray, optical, and radio data obtained for GRB 061121 . The vertical dotted lines indicate the times used for the SED plots shown in Fig. 11, while the curved dotted lines show the fit to the X-ray and optical data, including a late-time break, as described in the text.

Figures 4 and 10 show that all three instruments on board Swift saw the prompt emission around $75 \mathrm{~s}$ after the BAT trigger. However, it is noticeable that most of the emission is in the gammaray and X-ray bands, with the optical showing a relatively small increase in brightness in comparison. Assuming that the observed process is synchrotron, then the prompt emission that $i s$ detected by the UVOT will be the low-frequency extension of this in the internal shock. No reverse shock is apparent.

\subsection{Afterglow Emission}

\subsubsection{Broken Power-Law Decline Models}

The afterglow of GRB 061121 was observed over an even broader energy range (from radio to X-rays) than the prompt emission, with multicolor data being obtained from $\sim 100$ to $10^{5} \mathrm{~s}$ after the trigger. The X-ray light curve shows evidence for substantial curvature at later times (see Fig. 3), as has been found for other Swift GRBs (e.g., GRB 050315, Vaughan et al. 2006; GRB 060614, Gehrels et al. 2006b). The standard practice has been to fit such a decay using a series of power-law segments as a function of time. An alternative exponential-to-power-law description of the light curve is given in $\S$ 3.3.2.

Nousek et al. (2006) and Zhang et al. (2006) have both discussed the canonical shape that many Swift afterglows seem to follow: steep to plateau to shallow, with some light curves showing a further steepening. In these previous works, the extrapolation of the BAT data into the XRT band was incorporated into the derivation of the steep decay at the start of the canonical lightcurve shape. In the case of GRB 061121, the full curve can be seen entirely in X-rays, suggesting that the previous extrapolations are reliable. For the afterglow of GRB 061121, only data after the end of the main burst have been modeled with power laws. The early steep decline, which might be attributable to the curvature effect (Kumar \& Panaitescu 2000; Dermer 2004; Fan $\&$ Wei 2005), is not considered here.

According to the model proposed in Nousek et al. (2006) and Zhang et al. (2006), the plateau phase of the light curve is due to energy injection in the fireball. The plateau phase of GRB 061121 is consistent with an injection of energy since the luminosity in$\operatorname{dex}, q$, is negative, which is the requirement for injection to 
modify the afterglow (Zhang et al. 2006); the later two stages both have $q>1$. However, as discussed in $\S 3.3 .2$, the plateau and final transition to the power-law decay are only visible in the X-ray data for GRB 061121; the start of the final decay is much earlier in the $V$ and $R$ bands (see Fig. 10). One might expect that energy injection would affect all the energy bands simultaneously, rather than just the X-rays.

From the standard afterglow model computations (e.g., Zhang \& Mészáros 2004), we find that none of the closure relations fit the entire data set completely: although the shallow phase (after the end of energy injection, between $T+2.3 \mathrm{ks}$ and $T+32 \mathrm{ks}$ ) could be consistent with the evolution of a blast wave that had already entered the slow-cooling regime when deceleration started [i.e., $\nu>\max \left(\nu_{m}, \nu_{c}\right)$, where $\nu_{c}$ is the cooling frequency and $\nu_{m}$ is the synchrotron injection frequency; Sari et al. 1998; Chevalier $\&$ Li 2000], the steeper part of the decay curve $(T>32 \mathrm{ks})$ is not consistent with any of the models. This lack of consistency suggests that a different approach is required.

The change in decay slope between the shallow to steep phases ( $\sim 32 \mathrm{ks}$ ) cannot be easily identified with a jet break. It certainly seems unlikely that the simplest side-spreading jet model could be applicable, since the postbreak decay index $(\alpha \sim 1.5)$ is not steep enough (a postjet decay has $\alpha=p$, where $p$ is the electron index). There is also some indication that the X-ray spectral slope hardens after the break, whereas no change in spectral signature is expected over a jet break. In the case of a nonlaterally expanding jet (Panaitescu \& Mészáros 1999), $\alpha=(3 \beta / 2)+$ 0.25 (for a homogeneous circumstellar medium [CSM]; Panaitescu et al. 2006b), which does, indeed, fit the data after this break: $[1.5 \times(0.9 \pm 0.08)]+0.25=1.6 \pm 0.1$; the measured $\alpha$ is 1.53 . Such a confined jet has been suggested as an explanation for the observed decay in a number of previous bursts (e.g., GRB 990123, Kulkarni et al. 1999; GRB 050525A, Blustin et al. 2006; GRB 061007, Schady et al. 2006). The UVOT data obtained around this time show little evidence for a break, whereas jet breaks should occur across all energy bands simultaneously. However, nonsimultaneity could be explained by a multicomponent outflow, where the X-ray emission is produced within a narrow jet, while the optical component comes from a wider jet with lower Lorentz factor (Panaitescu \& Kumar 2004; Oates et al. 2007). There remains the issue, however, that $\alpha$ should steepen by 0.75 over a jet break (Mészáros \& Rees 1999), whereas the maximum observed change (within the $90 \%$ errors) is only $\Delta \alpha<0.61$, excluding $\Delta \alpha=0.75$ at almost $3 \sigma$; also, again there should be no spectral evolution across the break. There is, however, a probable jet break at later times, which is covered in the next section.

Other multicomponent models (see, e.g., Oates et al. 2007 and references therein) also fail to explain the data because of the lack of observed energy injection (plateau phase) in the optical data.

Panaitescu et al. (2006a) discuss chromatic breaks in Swift light curves and postulate that these could be due to a change in microphysical parameters within a wind environment. However, this model requires the cooling frequency to lie between the $\mathrm{X}$-ray and optical bands and, as is discussed in $\S 3.3 .2$, this does not seem to be the case here.

\subsubsection{Exponential to Power-Law Decline Model}

As first described by O'Brien et al. (2006) and further expanded by Willingale et al. (2007), GRB light curves can be well modeled by one or two components comprised of an early exponential rise followed by a power-law decay phase. Of these components, the first represents the prompt gamma-ray emission and early X-ray decay. The second, when detected, dominates at later times, forming what we see as the afterglow. These results show that fitting an intrinsically curved decay with multiple power-law segments runs the risk of incorrectly identifying temporal breaks (see also T. Sakamoto et al. 2007, in preparation). In this section the models of O'Brien et al. (2006) and Willingale et al. (2007) are applied to the multiband afterglow data of GRB 061121.

Figure 10 brings together the BAT, XRT, UVOT, FTN, and ROTSE data, along with further optical and radio points taken from the GCN Circulars (Halpern et al. 2006a, 2006b; Halpern \& Armstrong 2006a, 2006b; Chandra \& Frail 2006; van der Horst et al. 2006a, 2006b) and the upper limit from Chandra, to form a multienergy decay plot. The data have been plotted as "time since trigger $+4 \mathrm{~s}$ " in order to include the precursor on a $\log$ timescale. The optical points have all been corrected for extinction using $A_{V}=1.2$ (a combination of the Galactic value of 0.151 and an estimate of $A_{V} \sim 1$ for the GRB host galaxy; see $\S 2.4 .2$ ).

The contribution from the host galaxy reported by Malesani et al. (2006) and Cobb (2006) has been subtracted from the $V$ - and $R$-band flux values. The magnitude of the host in the $V$ band is 22.4 , which only changes the last two or three $V$-band points by a small amount. For the $R$ band we have no direct measurement, but the last group of MDM exposures gave an $R$ magnitude of 22.7 , corresponding to a flux level of $2.8 \mu \mathrm{Jy}$, and the flux level is still declining at that epoch $\left(\sim 3.3 \times 10^{5} \mathrm{~s}\right)$, so an $R$-band flux level of $2.5 \mu \mathrm{Jy}$ was adopted for the host. The error bars shown on the last few points reflect the large uncertainty in the galaxy contribution subtracted.

The curved dotted lines in Figure 10 are the fits to the data using the exponential-to-power-law model, followed by a break to a steeper decay around $10^{5} \mathrm{~s}$. These models are parameterized by the power-law decay, $\alpha$, and $T_{a}$, the time at which this decay is established. For the X-ray data, $T_{a, \mathrm{X}}$ is found to be $5250_{-460}^{+500} \mathrm{~s}$ and $\alpha_{a, \mathrm{X}}=1.32 \pm 0.03$. Fits were also performed to the $\bar{V}_{-}^{460}$ and $R$-band data, yielding $\alpha_{a, V}=0.66 \pm 0.04$ (with $T_{a, V}=70_{-70}^{+60} \mathrm{~s}$ ) and $\alpha_{a, R}=0.84 \pm 0.03\left(T_{a, R}=230_{-230}^{+120} \mathrm{~s}\right)$.

The nondetection by Chandra almost 2 months after the burst shows that there must have been a further steepening in the X-ray regime, and the optical data are not inconsistent with this finding. Constraining the temporal index after the late break to be $\alpha=2$ (a typical slope for a post-jet break decay), break times of $\sim 2.5 \times$ $10^{5}, \sim 2.5 \times 10^{4}$, and $\sim 10^{5} \mathrm{~s}$ are estimated for the X-ray, $V$ band, and $R$ band, respectively; note that the UVOT $V$-band value is particularly uncertain, given the small number of data points at late times. Within the uncertainties, these times are likely to be consistent, so the turnover could be achromatic, as required for a jet break. From Willingale et al. (2007), a jet break might be expected at $\sim 100 T_{a, \mathrm{X}}$, i.e., $5.5 \times 10^{5} \mathrm{~s}$, which is in agreement with these fits.

As can be seen from these numbers and the models plotted in Figure 10, the X-ray data clearly show the transition from the plateau to the power-law decay, whereas the start of the final decay is much earlier in the $V$ and $R$ bands. The $V$-band decay is also significantly flatter (by $\alpha \sim 0.2$ ) than that estimated for the $R$ band. As previously stated, the $V, B$, and $U$ light curves are all consistent with this slow decay. There have been few multicolor optical decay curves obtained for GRB afterglows, and, of these, the different filters (in the case of GRB 061007 [Schady et al. 2006; Mundell et al. 2007] X-ray and gamma-ray data, as well as the optical) tend to track each other (e.g., Guidorzi et al. 2005; Blustin et al. 2006; de Ugarte Postigo et al. 2007). In the case of GRB 061121, we find that the $R$-band data are fading more rapidly than the $V$. GRB 060218 , which was associated with a supernova (e.g., Campana et al. 2006b), shows changes throughout the optical spectra because of a combination of shock breakout and radioactive heating of the supernova ejecta. There is a large 


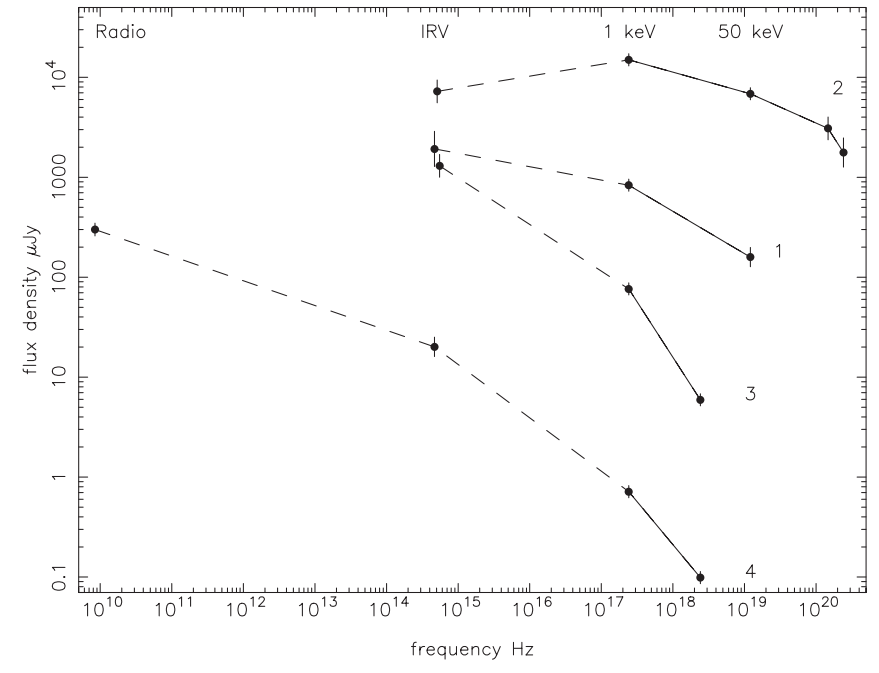

FIG. 11.- SEDs for the four time intervals indicated in Fig. 10. SED 2 (the peak of the burst emission) includes the Konus-Wind data, although these have not been included in Fig. 10. The solid lines represent the power-law fits to the BAT, XRT, and Konus data, while the dashed lines join the radio, optical, and $1 \mathrm{keV}$ points. Spectral evolution over time is clearly seen.

difference between the decays of the blue $(V, U, B)$ and red $(R)$ data for GRB 061121, which cannot be easily explained by a synchrotron spectrum. Although no supernova has been detected in this case, we speculate that some form of presupernova thermal emission could possibly be affecting the optical data, adding energy into the blue end of the spectrum, thus slowing its decline.

After the break in the decays around $10^{5} \mathrm{~s}$, the light curves across all bands become more consistent with one another, although there are only limited data at such a late time.

The vertical dotted lines in Figure 10 show the times of the SEDs plotted in Figure 11; again, all points were corrected for an extinction of $A_{V}=1.2$, so that they represent the true SEDs (with the frequency in the observer's frame). The solid lines represent actual fits to the X-ray and gamma-ray data, while the dashed lines just join the separate radio, optical, and $1 \mathrm{keV}$ points. The times of these SEDs, which clearly show spectral evolution, correspond to (1) before the main BAT peak, $56 \mathrm{~s}$ after trigger; (2) at the BAT peak, $76 \mathrm{~s}$ after trigger; (3) just after the start of the plateau, $300 \mathrm{~s}$ after the trigger; and (4) in the main decay at $65 \mathrm{ks}$ (chosen because radio measurements were taken at this time). SEDs 3 and 4 do not contain any BAT or Konus data, since the gamma-ray flux had decayed by this point; the highest energy point in these corresponds to the maximum energy $(10 \mathrm{keV})$ of the X-ray fits.
Table 4 demonstrates that the optical and X-ray spectra during the peak emission are best fitted with a broken power-law model, with the break energy at the very low energy end of the X-ray bandpass. SED 2 in Figure 11 shows that this spectral break corresponds to the peak frequency in a flux density plot ( $\beta_{1}$ is less than zero in this case). Only during SED 2 is the optical flux density lower than that of the higher energy data. Figure 4 also shows that the optical emission is less strong than the X-ray and gammaray data during the main burst.

Table 5 shows the values of $\alpha$ for the X-ray and optical decays (i.e., before and after the break) in SED 4, at $65 \mathrm{ks}$, with their corresponding spectral indices. For the initial stages of the powerlaw decay $\left(T_{a}<t<65,000 \mathrm{~s}\right)$ the evolution of the afterglow SED and the coupling between the temporal and spectral indices are not completely consistent with the standard model: although the $R$-band decay, with $\alpha_{a, R}=0.84 \pm 0.03$, is in good agreement with the homogeneous CSM model below the cooling break, the $\mathrm{X}$-ray and $V$-band flux decays are slower than expected from the measured spectral indices; they are in best agreement with the same constant density model below $\nu_{c}$, however.

The point at which the power-law decay dominates the exponential in the optical bands is noticeably earlier than in the X-ray (less than a few hundred seconds, rather than $\sim 5000 \mathrm{~s}$ ), and, as mentioned above, the decay indices are significantly different for all three (X-ray, $V$, and $R$ ) bands (see Fig. 10). At the time of SED 3, the X-ray data are not decaying (i.e., this is during the plateau), yet both the $V$ - and $R$-band data have already entered the power-law decline phase. The $R$ band is decaying faster than the $V$ band, so the spectral index through the optical range is becoming harder. The X-ray spectral index shows a similar hardening trend (see Table 3), so the SED measured from optical to $10 \mathrm{keV}$ is gradually getting harder. Such spectral hardening from the plateau to the final decay is a feature of many X-ray afterglows (Willingale et al. 2007).

This slow hardening of the broadband spectrum with time could be a signature of synchrotron self-Compton emission (Sari \& Esin 2001; Panaitescu \& Kumar 2000). The strength of the self-Compton component in the afterglow depends on the flux of low-energy photons (radio-optical) and the electron density in the shock. Using the formulation in Sari \& Esin (2001), the density required is given by

$$
n_{1}=3 \times 10^{9}\left(\frac{f_{\max }^{\mathrm{IC}}}{f_{\max }^{\text {sync }}}\right)^{4 / 3}\left(E_{52} t_{\text {day }}\right)^{-1 / 3} \mathrm{~cm}^{-3}
$$

where $f_{\max }^{\mathrm{IC}} / f_{\max }^{\mathrm{sync}}$ is the ratio of the peak flux of the seed synchrotron spectrum (i.e., the source of low-energy photons) and the peak flux of the self-Compton emission, $E_{52}$ is the isotropic burst

TABLE 5

Closure Relations

\begin{tabular}{|c|c|c|c|c|c|c|}
\hline GRB Models & $\alpha(\beta)$ & $\alpha\left(\beta_{a, \mathrm{X}}\right)^{\mathrm{b}}$ & $\alpha_{a, \mathrm{X}}^{\mathrm{a}}$ & $\alpha\left(\beta_{\text {opt }}\right)^{\mathrm{b}}$ & \multicolumn{2}{|c|}{$\alpha_{\text {opt }}^{\mathrm{a}}$} \\
\hline 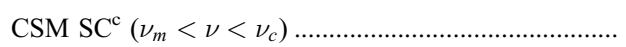 & $3 \beta / 2$ & $1.49 \pm 0.10$ & $1.32 \pm 0.03$ & $0.80 \pm 0.09$ & $0.66 \pm 0.04$ & $0.84 \pm 0.03$ \\
\hline Wind $\mathrm{SC}^{\mathrm{c}}\left(\nu_{m}<\nu<\nu_{c}\right)$ & $(3 \beta+1) / 2$ & $1.99 \pm 0.10$ & $1.32 \pm 0.03$ & $1.30 \pm 0.09$ & $0.66 \pm 0.04$ & $0.84 \pm 0.03$ \\
\hline $\mathrm{CSM}$ or Wind $\mathrm{SC}^{\mathrm{c}}$ and $\mathrm{FC}^{\mathrm{d}}\left[\nu>\max \left(\nu_{c}, \nu_{m}\right)\right] \ldots \ldots \ldots \ldots$ & $(3 \beta-1) / 2$ & $0.99 \pm 0.10$ & $1.32 \pm 0.03$ & $0.30 \pm 0.09$ & $0.66 \pm 0.04$ & $0.84 \pm 0.03$ \\
\hline
\end{tabular}

Note.-Closure relations for exponential plus power-law model fits to the X-ray data $\left(\beta_{a, \mathrm{X}}=0.99 \pm 0.07\right)$ and the optical to X-ray band $\left(\beta_{\mathrm{opt}}=0.53 \pm 0.06\right)$ from the time of SED 4 (65 ks after the burst).

${ }^{a}$ Observed power-law decay index.

${ }^{b}$ Decay calculated from the measured spectral index.

c Slow cooling.

d Fast cooling. 
energy in units of $10^{52} \mathrm{ergs}$, and $t_{\text {day }}$ is the time in days after the burst (which determines the distance through the CSM swept up by the external shock). From Figure 11 (SEDs 1, 3, and 4) we see that $f_{\max }^{\mathrm{IC}} / f_{\max }^{\text {sync }} \sim 0.001$ if the X-ray flux has a significant contribution from a self-Compton component at $t_{\text {day }}=0.75$. A value of $E_{52}=30$ gives $n_{1} \approx 10^{5} \mathrm{~cm}^{-3}$. Even assuming that the emission at 0.75 days is not dominated by the self-Comptonization, and so taking the $f_{\max }^{\mathrm{IC}} / f_{\max }^{\mathrm{sync}}$ ratio to be a factor of 10 smaller, the density would be $\sim 5 \times 10^{3} \mathrm{~cm}^{-3}$, which is still high. It seems unlikely that self-Compton emission is the cause of the spectral hardening of the SED unless the CSM density encountered by the external shock is extremely large. However, there have been suggestions that GRBs may form in molecular clouds (Galama \& Wijers 2001; Campana et al. 2006a, 2007), which have densities of $10^{4}$ or more particles per cubic centimeter in the cores (Miyazaki \& Tsuboi 1999; Wilson et al. 1999). Typically one might expect greater reddening than is found here (Table 4), although Waxman \& Draine (2000) discuss the possibility of dust destruction.

The spectrum will be redshifted as the jet slows down, so the optical and X-ray spectral indices should, if anything, become softer, the opposite of what is seen here. Although spectral hardening with time is suggested from the data, it is not easily explained by current models.

Whether or not there is a Comptonized component, the later SEDs clearly indicate that there is a break in the spectrum somewhere between the optical and the X-ray; this is also shown by the fits in Table 4, where the UVOT/XRT spectra are better fitted with broken power laws, with $E_{\text {break }}$ toward the low-energy end of the X-ray bandpass. Since both the optical and X-ray bands appear to be below the cooling frequency, from the closure relations given in Table 5, this change in slope cannot be identified with a cooling break; its origin remains unclear.

The redshift of $z=1.314$ and the isotropic energy of $E_{\text {iso }} \sim 3 \times$ $10^{53}$ ergs $(\S 2.4 .1)$ can be used to place constraints on the jet opening angle. From Sari et al. (1999), and assuming that the jet break occurs at $T_{0}+2 \times 10^{5} \mathrm{~s}$, we have $\theta_{j} \sim 4^{\circ}\left(\eta_{\gamma} / 0.2\right)^{1 / 8}(n / 0.1)^{1 / 8}$, where $n$ and $\eta_{\gamma}$ are the density of the CSM and the efficiency of the fireball in converting the energy in the ejecta into gamma rays, respectively. Taking $\eta_{\gamma}=0.2$ and $n=3 \mathrm{~cm}^{-3}$ (following Ghirlanda et al. 2004), this gives $E_{\gamma} \sim 1.7 \times 10^{51}$ ergs for the beaming-corrected gamma-ray energy released, which is within the range previously determined (e.g., Frail et al. 2001) and consistent with the Ghirlanda relationship (Ghirlanda et al. 2004).

\section{SUMMARY AND CONCLUSIONS}

Swift triggered on a precursor to GRB 061121, leading to unprecedented coverage of the prompt emission by all three instru- ments on board, with the gamma-ray, X-ray, and optical/UV bands all tracking the main peak of the burst. GRB 061121 is the instantaneously brightest long Swift burst detected thus far, in both gamma rays and X-rays. The precursor and main burst show spectral lags of different lengths, although both are consistent with the lag-luminosity relation for long GRBs (Gehrels et al. 2006b).

The SED of the prompt emission, stretching from $1 \mathrm{eV}$ to $1 \mathrm{MeV}$, shows a peak flux density at around $1 \mathrm{keV}$ and is harder than the standard model predicts. There is definite curvature in the spectra, with the prompt optical to X-ray spectrum being better fitted by a broken power law, similar to results found for fitting X-ray flares (e.g., Guetta et al. 2007; Goad et al. 2007).

The afterglow component, in both the optical and X-ray, starts early on, before or during the main burst peak (see also O'Brien et al. 2006; Willingale et al. 2007; Zhang et al. 2007). The broadband SEDs reveal gradual spectral hardening as the afterglow evolves, both within the X-ray regime ( $\Gamma$ flattening from $\sim 2.05$ to $\sim 1.87)$ and between the $V$ - and $R$-band optical data ( $\alpha_{V} \sim 0.66$ compared with $\alpha_{R} \sim 0.84$ ). Self-Comptonization could explain the hardening, although a molecular cloud core density would be required. A probable jet break occurs around $T_{0}+2 \times 10^{5} \mathrm{~s}$, shown by a late-time nondetection by Chandra. Before this break, the $\mathrm{X}$-ray and $V$-band decays are too slow to be readily explained by the standard models.

This extremely well sampled burst shows clearly that there remains much work to be done in the field of GRB models. A single, unified model for all GRB emission observed should be the ultimate goal.

The authors gratefully acknowledge support for this work at the University of Leicester by PPARC, at PSU by NASA, and in Italy by funding from ASI. This work is partly based on observations with the Konus-Wind experiment (supported by the Russian Space Agency contract and RFBR grant 06-02-16070) and on data obtained with XMM-Newton, an ESA science mission with instruments and contributions directly funded by ESA Member States and NASA. We thank the Liverpool GRB group at ARI, Liverpool John Moores University, in particular C. J. Mottram, D. Carter, R. J. Smith, and A. Gomboc, for their assistance with the FTN data acquisition and interpretation. The Faulkes Telescopes are operated by the Las Cumbres Observatory Global Telescope Network. We also thank J. E. Hill and A. F. Abbey for discussions and help with the PuPD data, P. Curran for assistance with the UVOT/XRT SED creation, D. Grupe for the Chandra upper limit, and B. Cobb and D. Malesani for information regarding the magnitude of the host galaxy. Thanks as well to C. Akerlof, E. Rykoff, A. Phillips, and M. C. B. Ashley from the ROTSE team.

\section{REFERENCES}

Abbey, A. F., et al. 2005, in The X-Ray Universe 2005, ed. A. Wilson (ESA-SP 604; Noordwijk: ESA), 943

Akerlof, C., et al. 1999, Nature, 398, 400

Amati, L., et al. 2002, A\&A, 390, 81

Aptekar, R. L., et al. 1995, Space Sci. Rev., 71, 265

Band, D., et al. 1993, ApJ, 413, 281

Barthelmy, S. D., et al. 2005, Space Sci. Rev., 120, 143

Bellm, E., Bandstra, M., Boggs, S., Wigger, C., Hajdas, W., Smith, D. M., \& Hurley, K. 2006, GCN Circ. 5838, http://gcn.gsfc.nasa.gov/gcn/gcn3/5838.gcn3 Blake, C. H., et al. 2005, Nature, 435, 181

Bloom, J. S., Frail, D. A., \& Kulkarni, S. R. 2003, ApJ, 594, 674

Bloom, J. S., Perley, D. A., \& Chen, H. W. 2006, GCN Circ. 5826, http://gen .gsfc.nasa.gov/gen $/ g \mathrm{gcn} 3 / 5826 . \mathrm{gcn} 3$

Blustin, A. J., et al. 2006, ApJ, 637, 901
Boër, M., Atteia, J. L., Damerdji, Y., Gendre, B., Klotz, A., \& Stratta, G. 2006, ApJ, 638, L71

Borgonovo, L., \& Ryde, F. 2001, ApJ, 548, 770

Bouchet, P., Lequeux, J., Maurice, E., Prevot, L., \& Prevot-Burnichon, M. L. 1985, A\&A, 149, 330

Burrows, D. N., et al. 2005a, Science, 309, 1833

. 2005b, Space Sci. Rev., 120, 165

Campana, S., et al. 2006a, A\&A, 449, 61 2006b, Nature, 442, 1008 2007, ApJ, 654, L17

Cardelli, J. A., Clayton, G. C., \& Mathis, J. S. 1989, ApJ, 345, 245

Cenko, S. B. 2006, GCN Circ. 5844, http://gcn.gsfc.nasa.gov/gen/gcn3/5844 .gen 3

Cenko, S. B., et al. 2006, ApJ, 652, 490 
Chandra, P., \& Frail, D. A. 2006, GCN Circ. 5843, http://gcn.gsfc.nasa.gov/ $\mathrm{gcn} / \mathrm{gcn} 3 / 5843$.gcn 3

Cheng, L. X., Ma, Y. Q., Cheng, K. S., Lu, T., \& Zhou, Y. Y. 1995, A\&A, 300, 746

Chester, M., Page, M., Roming, P., Marshall, F., Boyd, P., Angelini, L., Greiner, J., \& Gehrels, N. 2005, GCN Circ. 3838, http://gen.gsfc.nasa.gov/gcn/gen3/ 3838.gcn 3

Chevalier, R. A., \& Li, Z.-Y. 2000, ApJ, 536, 195

Cobb, B. E. 2006, GCN Circ. 5878, http://gcn.gsfc.nasa.gov/gcn/gcn3/5878.gcn3

Cummings, J., et al. 2005, GCN Circ. 3835, http://gcn.gsfc.nasa.gov/gcn/gcn3/ 3835.gcn 3

den Herder, J. W., et al. 2001, A\&A, 365, L7

Dermer, C. D. 2004, ApJ, 614, 284

de Ugarte Postigo, A., et al. 2007, A\&A, 462, L57

Dickey, J. M., \& Lockman, F. J. 1990, ARA\&A, 28, 215

Diplas, A., \& Savage, B. D. 1994, ApJ, 427, 274

Efimov, Yu., Rumyantsev, V., \& Pozanenko, A. 2006a, GCN Circ. 5850, http:// gcn.gsfc.nasa.gov/gcn $/ \mathrm{gcn} 3 / 5850 . \mathrm{gcn} 3$

. 2006b, GCN Circ. 5870, http://gcn.gsfc.nasa.gov/gcn/gcn3/5870.gcn3

Fan, Y. Z., \& Wei, D. M. 2005, MNRAS, 364, L42

Fenimore, E. E., in't Zand, J. J. M., Norris, J. P., Bonnell, J. T., \& Nemiroff, R. J. 1995, ApJ, 448, L101

Fenimore, E. E., \& Ramirez-Ruiz, E. 1999, ApJ, submitted (astro-ph/9909299)

Ford, L. A., et al. 1995, ApJ, 439, 307

Frail, D. A., et al. 2001, ApJ, 562, L55

Fukugita, M., Shimasaku, K., \& Ichikawa, T. 1995, PASP, 107, 945

Galama, T. J., \& Wijers, R. A. M. J. 2001, ApJ, 549, L209

Gehrels, N., Page, K., Barthelmy, S., Burrows, D. N., Marshall, F., Roming, R., Sakamoto, T., \& Sato, G. 2006a, GCN Circ. 5839, http://gcn.gsfc.nasa.gov/ $\mathrm{gcn} / \mathrm{gcn} 3 / 5839$.gcn 3

Gehrels, N., et al. 2004, ApJ, 611, 1005 2006b, Nature, 444, 1044

Ghirlanda, G., Ghisellini, G., \& Lazzati, D. 2004, ApJ, 616, 331

Ghisellini, G., Celotti, A., \& Lazzati, D. 2000, MNRAS, 313, L1

Goad, M. R., et al. 2007, A\&A, in press (astro-ph/0612661)

Golenetskii, S., Aptekar, R., Mazets, E., Pal'shin, V., Frederiks, D., \& Cline, T. 2006, GCN Circ. 5837, http://gcn.gsfc.nasa.gov/gen/gen3/5837.gcn3

Golenetskii, S. V., Mazets, E. P., Aptekar, R. L., \& Ilinskii, V. N. 1983, Nature, 306,451

Guetta, D., et al. 2007, A\&A, 461, 95

Guidorzi, C., et al. 2005, ApJ, 630, L121

Hakkila, J., \& Giblin, T. W. 2004, ApJ, 610, 361

Halpern, J. P., \& Armstrong, E. 2006a, GCN Circ. 5851, http://gcn.gsfc.nasa .gov/gen/gen $3 / 5851 . g \mathrm{cn} 3$

2006b, GCN Circ. 5853, http://gcn.gsfc.nasa.gov/gen/gen3/5853.gcn3

Halpern, J. P., Mirabal, N., \& Armstrong, E. 2006a, GCN Circ. 5840, http:// gen.gsfc.nasa.gov/gen $/ \mathrm{gcn} 3 / 5840 . \mathrm{gcn} 3$

2006b, GCN Circ. 5847, http://gcn.gsfc.nasa.gov/gcn/gcn3/5847.gcn3

Hill, J. E., et al. 2004, Proc. SPIE, 5165, 217

2006, ApJ, 639, 303

in't Zand, J. J. M., \& Fenimore, E. E. 1996, ApJ, 464, 622

Jansen, F., et al. 2001, A\&A, 365, L1

Klotz, A., Gendre, B., Stratta, G., Atteia, J. L., Boër, M., Malacrino, F., Damerdji, Y., \& Behrend, R. 2006, A\&A, 451, L39

Koornneef, J. 1982, A\&A, 107, 247

Kulkarni, S. R., et al. 1999, Nature, 398, 389

Kumar, P., \& Panaitescu, A. 2000, ApJ, 541, L51

Lazzati, D. 2005, MNRAS, 357, 722

Lazzati, D., Morsony, B. J., \& Begelman, M. C. 2007, Philos. Trans. R. Soc. London A, 365, 1141

Liang, E. W., et al. 2006, ApJ, 646, 351

Link, B., Epstein, R. I., \& Priedhorsky, W. C. 1993, ApJ, 408, L81

Lloyd, N. M., \& Petrosian, V. 2000, ApJ, 543, 722

Madau, P. 1995, ApJ, 441, 18

Madau, P., Ferguson, H. C., Dickinson, M. E., Giavalisco, M., Steidel, C. C., \& Fruchter, A. 1996, MNRAS, 283, 1388

Malesani, D., et al. 2006, GCN Circ. 5877, http:/gcn.gsfc.nasa.gov/gcn/gcn3/ 5877.gcn 3

Marshall, F. E. 2006, GCN Circ. 5899, http://gcn.gsfc.nasa.gov/gen/gen3/5899 gcn 3

Marshall, F. E., Holland, S. T., \& Page, K. L. 2006, GCN Circ. 5833, http:// gen.gsfe.nasa.gov/gen/gen3/5833.gen3

Medvedev, M. V. 2000, ApJ, 540, 704

Melandri, A., et al. 2006, GCN Circ. 5827, http:/gcn.gsfc.nasa.gov/gcn/gcn3/ 5827.gcn 3

Mészáros, P., \& Rees, M. J. 1999, MNRAS, 306, L39

Mészáros, P., Rees, M. J., \& Papathanassiou, H. 1994, ApJ, 432, 181

Miyazaki, A., \& Tsuboi, M. 1999, Adv. Space Res., 23, 973

Mundell, C. G., et al. 2007, ApJ, 660, 489
Nakamura, T. 2000, ApJ, 534, L159

Norris, J. P. 2002, ApJ, 579, 386

Norris, J. P., \& Bonnell, J. T. 2006, ApJ, 643, 266

Norris, J. P., Marani, G. F., \& Bonnell, J. T. 2000, ApJ, 534, 248

Norris, J. P., Nemiroff, R. J., Bonnell, J. T., Scargle, J. D., Kouveliotou, C.,

Paciesas, W. S., Meegan, C. A., \& Fishman, G. J. 1996, ApJ, 459, 393

Nousek, J. A., et al. 2006, ApJ, 642, 389

Oates, S. R., et al. 2007, MNRAS, submitted

O’Brien, P. T., et al. 2006, ApJ, 647, 1213

Paciesas, W. S., et al. 1999, ApJS, 122, 465

Page, K. L., Beardmore, A. P., Goad, M. R., Kennea, J. A., Burrows, D. N., Marshall, F., \& Smale, A. 2005a, GCN Circ. 3837, http://gcn.gsfc.nasa.gov/ $\mathrm{gcn} / \mathrm{gcn} 3 / 3837 . \mathrm{gcn} 3$

Page, K. L., Starling, R. L. C., Osborne, J. P., Troja, E., \& Morris, D. 2006a, GCN Circ. 5832, http://gcn.gsfc.nasa.gov/gcn/gcn3/5832.gcn3

Page, M., et al. 2005b, GCN Circ. 3830, http://gcn.gsfc.nasa.gov/gen/gcn3/3830 gcn3

- 2006b, GCN Circ. 5823, http://gcn.gsfc.nasa.gov/gen/gen3/5823.gen3

Panaitescu, A., \& Kumar, P. 2000, ApJ, 543, 66

2004, MNRAS, 350, 213

2007, MNRAS, 376, 1065

Panaitescu, A., \& Mészáros, P. 1999, ApJ, 526, 707

Panaitescu, A., Mészáros, P., Burrows, D., Nousek, J., Gehrels, N., O’Brien, P., \& Willingale, R. 2006a, MNRAS, 369, 2059

Panaitescu, A., Mészáros, P., Gehrels, N., Burrows, D., \& Nousek, J. 2006b, MNRAS, 366, 1357

Pe'er, A., Mészáros, P., \& Rees, M. J. 2005, ApJ, 635, 476

Pe'er, A., \& Zhang, B. 2006, ApJ, 653, 454

Pei, Y. C. 1992, ApJ, 395, 130

Perley, D. A., \& Bloom, J. S. 2006, GCN Circ. 5825, http://gcn.gsfc.nasa.gov/ $\mathrm{gcn} / \mathrm{gcn} 3 / 5825 . \mathrm{gcn} 3$

Pilla, R. P., \& Loeb, A. 1998, ApJ, 494, L167

Piro, L., et al. 1998, A\&A, 329, 906

Ramirez-Ruiz, E., Celotti, A., \& Rees, M. J. 2002a, MNRAS, 337, 1349

Ramirez-Ruiz, E., MacFadyen, A. I., \& Lazzati, D. 2002b, MNRAS, 331, 197

Rees, M. J., \& Mészáros, P. 2005, ApJ, 628, 847

Romano, P., et al. 2006, A\&A, 456, 917

Roming, P. W. A., et al. 2005, Space Sci. Rev., 120, 95

Sari, R., \& Esin, A. A., 2001, ApJ, 548, 787

Sari, R., \& Piran, T. 1999, ApJ, 520, 641

Sari, R., Piran, T., \& Halpern, J. P. 1999, ApJ, 519, L17

Sari, R., Piran, T., \& Narayan, R. 1998, ApJ, 497, L17

Schady, P., et al. 2006, MNRAS, submitted (astro-ph/0611081) 2007, MNRAS, 377, 273

Schartel, N. 2006, GCN Circ. 5829, http://gcn.gsfc.nasa.gov/gcn/gcn3/5829.gcn3

Schlegel, D. J., Finkbeiner, D. P., \& Davis, M. 1998, ApJ, 500, 525

Sonoda, E., Maeno, S., Hara, T., Matsumura, T., Tanaka, K., Tanaka, T., \& Yamauchi, M. 2006, GCN Circ. 5830, http://gcn.gsfc.nasa.gov/gen/gcn3/ $5830 . \mathrm{gcn} 3$

Starling, R. L. C., Wijers, R. A. M. J., Wiersema, K., Rol, E., Curran, P. A., Kouveliotou, C., van der Horst, A. J., \& Heemskerk, M. H. M. 2007, ApJ, in press (astro-ph/0610899)

Strüder, L., et al. 2001, A\&A, 365, L18

Tang, S. M., \& Zhang, S. N. 2006, A\&A, 456, 141

Torii, K. 2006, GCN Circ. 5845, http://gcn.gsfc.nasa.gov/gcn/gcn3/5845.gcn3

Turner, M. J. L., et al. 2001, A\&A, 365, L27

Uemura, M., Arai, A., \& Uehara, T. 2006, GCN Circ. 5828, http://gcn.gsfc nasa.gov/gen/gen $3 / 5828$.gcn3

Umeda, H., Tominaga, N., Maeda, K., \& Nomoto, K. 2005, ApJ, 633, L17

van der Horst, A. J., Wijers, R. A. M. J., \& Rol, E. 2006a, GCN Circ. 5871, $\mathrm{http} / / / \mathrm{gcn} . \mathrm{gsfc}$. nasa.gov/gen $/ \mathrm{gcn} 3 / 5871 . \mathrm{gcn} 3$ . 2006b, GCN Circ. 5874, http://gcn.gsfc.nasa.gov/gcn/gcn3/5874.gcn3

Vaughan, S., et al. 2006, ApJ, 638, 920

Vestrand, W. T., et al. 2005, Nature, 435, 178

2006, Nature, 442, 172

Waxman, E., \& Draine, B. T. 2000, ApJ, 537, 796

Waxman, E., \& Mészáros, P. 2003, ApJ, 584, 390

Willingale, R., et al. 2007, ApJ, in press

Wilms, J., Allen, A., \& McCray, R. 2000, ApJ, 542, 914

Wilson, T. L., Mauersberger, R., Gensheimer, P. D., Muders, D., \& Bieging, J. H. 1999, ApJ, 525, 343

Yost, S. A., Schaefer, B. E., \& Yuan, F. 2006, GCN Circ. 5824, http://gen .gsfc.nasa.gov/gcn/gcn $3 / 5824$.gcn3

Zhang, B., Fan, Y. Z., Dyks, J., Kobayashi, S., Mészáros, P., Burrows, D. N.,

Nousek, J. A., \& Gehrels, N. 2006, ApJ, 642, 354

Zhang, B., \& Mészáros, P. 2002, ApJ, 581, 1236 2004, Int. J. Mod. Phys. A, 19, 2385

Zhang, B.-B., Liang, E.-W., \& Zhang, B. 2007, ApJ, in press (astro-ph/0612246) 